\title{
Models of Goldstone gauginos
}

\author{
Daniele S. M. Alves, ${ }^{1,2, *}$ Jamison Galloway, ${ }^{1, \dagger}$ Matthew McCullough, ${ }^{3, \ddagger}$ and Neal Weiner ${ }^{1, \S}$ \\ ${ }^{1}$ Center for Cosmology and Particle Physics, Department of Physics, New York University, \\ New York, New York 10003, USA \\ ${ }^{2}$ Department of Physics, Princeton University, Princeton, New Jersey 08544, USA \\ ${ }^{3}$ Theory Division, CERN, 1211 Geneva 23, Switzerland \\ (Received 20 August 2015; published 14 April 2016)
}

\begin{abstract}
Models with Dirac gauginos are appealing scenarios for physics beyond the Standard Model. They have smaller radiative corrections to scalar soft masses, a suppression of certain supersymmetry (SUSY) production processes at the LHC, and ameliorated flavor constraints. Unfortunately, they are generically plagued by tachyons charged under the Standard Model, and attempts to eliminate such states typically spoil the positive features. The recently proposed "Goldstone gaugino" mechanism provides a simple realization of Dirac gauginos that is automatically free of dangerous tachyonic states. We provide details on this mechanism and explore models for its origin. In particular, we find SUSY QCD models that realize this idea simply and discuss scenarios for unification.
\end{abstract}

DOI: 10.1103/PhysRevD.93.075021

\section{INTRODUCTION}

The turn-on of the LHC has challenged a number of basic ideas about naturalness. While parameter regions for supersymmetry and other solutions to the hierarchy problem remain, large swaths of "natural" parameter space have been eliminated, causing us to rethink whether the weak scale is, itself, natural [1-6].

A great challenge for a number of these scenarios comes from the renormalization group flow. In the minimal supersymmetric standard model (MSSM), for instance, large stop masses feed into the Higgs soft masses quickly, meaning that the stop masses should be ideally generated at a low scale. At the same time, existing searches for gluinos have been pushing their masses up, which, in turn, exacerbate their corrections to the stops and Higgs soft masses.

A simple resolution of this could lie in a departure from the framework of the MSSM. One simple deviation is to break supersymmetry with Dirac gauginos [7-14], rather than Majorana. A remarkable effect of the presence of the Dirac gaugino mass is that it is "supersoft" [14] and contributes only a finite threshold to the soft scalar masses. As a consequence, there is no sizable running contributing to the Higgs mass, and even very heavy squarks (stops) would introduce only mild $1 \%-10 \%$ tuning [14].

\footnotetext{
*spier@nyu.edu

jamison.galloway@nyu.edu

matthew.mccullough@cern.ch

§neal.weiner@nyu.edu
}

Published by the American Physical Society under the terms of the Creative Commons Attribution 3.0 License. Further distribution of this work must maintain attribution to the author(s) and the published article's title, journal citation, and DOI.
Moreover, this supersoftness can result in dramatic phenomenological effects. The leading effect is to have gauginos which are naturally 5-10 times heavier than the scalar sector, which is at odds with conventional expectations from the MSSM. By allowing this, a number of other possibilities arise. First, because the gauginos are both heavy and Dirac, the production cross section for colored scalars is suppressed, weakening limits on them [15]. Heavy Dirac gauginos also weaken flavor and $C P$ constraints considerably [16], alleviating the supersymmetry (SUSY) flavor and $C P$ problems. These setups can naturally arise from a number of UV scenarios [7-59].

From this, it would seem that Dirac gauginos are a promising approach to solving the naturalness issues of supersymmetry. There are, however, a few problems [14]: the existence of the supersoft operator naturally is accompanied by a $B \mu$-type term, which induces a tachyon for either the scalar or pseudoscalar in the adjoint; RG running tends to spread the gauginos widely, leading to very heavy gluinos; and finally, the $D$-term quartic for the Higgs boson is naturally suppressed. ${ }^{1}$ A singlet coupling to $H_{u} H_{d}$ in the superpotential can ameliorate the lack of $D$-term quartics, and modifying assumptions about gaugino universality can compensate for the running, which pulls up the gluino. The $B \mu$ term, however, is quite robust, appearing ubiquitously in models that generate the Dirac gaugino mass. Consequently, any scenario that truly allows natural models of Dirac gauginos must first address this $B \mu$ problem. Addressing this would eliminate the last vestiges of objections to the Dirac gaugino scenario.

In [60] a solution to the $B \mu$ problem of Dirac gauginos was proposed, coined the Goldstone gaugino ( $\mathrm{GoGa}$ )

\footnotetext{
${ }^{1}$ See [52] for a detailed discussion.
} 
mechanism. There, the right-handed gaugino originates from a (pseudo-)Goldstone superfield of a spontaneously broken anomalous global symmetry. In the GoGa scenario, the dangerous operators are naturally absent, while the Dirac gaugino mass is still present and originates from a WessZumino-Witten (WZW) term. In this paper, we outline the generic features of the Goldstone gaugino mechanism (Sec. II), and present natural UV completions to the GoGa scenario, one strongly coupled in the context of supersymmetric QCD (SQCD) with $F=N$ flavors (Sec. III), and another one perturbative (Sec. IV). Finally, we discuss their implications for naturalness (Sec. V B), gauge coupling unification (Sec. VC), and collider phenomenology (Sec. V D). We conclude in Sec. VI.

\section{GOLDSTONE GAUGINOS}

The Goldstone gaugino mechanism is a realization of Dirac gauginos and has been described in [60]. Here we review the basics of Dirac gauginos as well as the GoGa mechanism, outlining its essential ingredients and how it addresses the $B \mu$ problem.

The Dirac gaugino mass operator, to which we will refer as the classic supersoft (CS) operator, marries the fermion in the gauge vector supermultiplet to the fermion in an adjoint chiral superfield $A$,

$$
\Delta W=\frac{\sqrt{2}}{M_{\mathrm{C}}} W^{\prime \alpha} W_{\alpha}^{i} A^{i},
$$

where $W_{\alpha}^{\prime}$ is a spurion superfield for a $\mathrm{U}(1)$ that acquires a $D$-term expectation value $\left\langle W_{\alpha}^{\prime}\right\rangle=\theta_{\alpha} D$. This operator generates a Dirac mass $m_{D}=D / M_{\mathrm{C}}$ for the fermions, and a mass for the real scalar $m_{a_{R}}=2 m_{D}$, while leaving the pseudoscalar massless. Note that this operator respects a shift symmetry under which the pseudoscalar shifts by a constant, $a_{I} \rightarrow a_{I}+\chi$.

The dangerous $B \mu$-like term arises from

$$
\Delta W=\frac{1}{2 M_{\mathrm{LT}}^{2}} W^{\prime \alpha} W_{\alpha}^{\prime} A^{i} A^{i}
$$

to which we will refer as the "lemon-twist" supersoft (LTS) operator [30]. It generates a mass squared with opposite sign for $a_{R}$ and $a_{I}$, i.e. $\delta m_{a_{R}}^{2}=-\delta m_{a_{I}}^{2}=D^{2} / M_{\mathrm{LT}}^{2}$. In principle, the theory as written need not be problematic. If the LTS operator gives a negative mass squared to $a_{R}$, then in combination with the classic operator it may yield no tachyons.

As a matter of practical course, however, this is not the case. If both (2.1) and (2.2) arise at the same loop order, then we naturally expect $m_{a_{I}} \sim 4 \pi m_{D}$, and the classic supersoft operator cannot cancel the tachyon from the lemon-twist operator. This is aggravated by the fact that the classic operator comes with a $g_{i}$ for the gauge group indexed by $i$, which further suppresses it relative to lemon twist. A solution involving large additional $F$-term scalar masses for the adjoint breaks supersoftness, and so the standard model (SM) superpartners obtain masses fixed by a UV-sensitive logarithm rather than just the finite threshold, leading back to the need for tuning as in the conventional MSSM. A superpotential mass $\Delta W=m_{A} A^{2}$, as the other simple alternative, is unappealing since its required size would render the SM gauginos almost purely Majorana [14,61].

The essential lemon-twist issue is easily seen in the context of the simplest toy model that gives rise to Dirac gaugino masses. Consider, in addition to the chiral SM adjoint $A$, messengers $T, \bar{T}$ which are fundamentals under the SM gauge group and charged under the U(1) that carries the $D$-term vacuum expectation value (VEV). With the superpotential

$$
W=\lambda \bar{T} A T+\mu \bar{T} T
$$

and the masses for the messenger scalars split by the $D$-term, $\mu^{2} \pm D$, one-loop diagrams generate both classic and lemon-twist operators with coefficients

$$
\begin{aligned}
& \frac{1}{M_{\mathrm{C}}}=\sqrt{2} \frac{g_{i} \lambda}{16 \pi^{2}} \frac{1}{\mu}, \\
& \frac{1}{M_{\mathrm{LT}}^{2}}=-\frac{\lambda^{2}}{16 \pi^{2}} \frac{1}{\mu^{2}},
\end{aligned}
$$

where $g_{i}$ is the appropriate SM gauge coupling. The problem seems intractable: since we may write both $W^{\prime} W A$ and $W^{2}$, no obvious symmetry is able to forbid the lemon-twist operator, $\left(W^{\prime} A\right)^{2}$.

Ideally, we would impose a symmetry $a_{I} \rightarrow a_{I}+\chi$ with $\chi=$ const, which would forbid lemon twist but allow the classic operator. This breaks supersymmetry, however, and while a useful symmetry to keep in mind, it is difficult to implement. ${ }^{2}$ If we elevate the shift parameter $\chi$ to a chiral superfield and impose a supershift symmetry $A \rightarrow A+\chi$, this necessarily forbids both the classic and the lemontwist terms.

There is, however, a simple solution: namely, the Goldstone gaugino mechanism [60], whose essential ingredients we shall now review.

In the GoGa mechanism, a shift symmetry forbidding the lemon-twist operator emerges from the spontaneous breaking of an approximate global symmetry, which is explicitly

\footnotetext{
${ }^{2}$ Interestingly, this symmetry would allow an operator in the Kähler potential $\int d^{4} \theta D_{\alpha} V^{\prime} W^{\alpha}\left(A+A^{\dagger}\right)$ [52] ("lime twist"), which is not supersoft and induces large two-loop contributions to the soft masses of MSSM scalars. This operator is not gauge invariant, however, but could plausibly still arise if $V^{\prime}$ is simply a spurion field, not representing any genuine gauge symmetry. Still, if the symmetry represented by $V^{\prime}$ could have been gauged (in the sense that anomalies cancel and no other gauge noninvariant terms are present), which is the usual case, it will not arise radiatively.
} 
violated by the SM gauge couplings. Moreover, the currents associated with the broken generators of the global symmetry have a mixed anomaly under the $\mathrm{SM}$ and the U(1) that carries the $D$-term VEV. This mixed anomaly turns out to be the source of the classic supersoft operator, identified with a WZW term [62]. In this realization, the right-handed gauginos originate from the Goldstones of the broken symmetry. In the limit $g \rightarrow 0$ the anomaly vanishes and so does the classic supersoft operator, consistent with the restoration of an exact shift symmetry.

A simple toy model illustrating this mechanism is the following. Consider as before the messenger sector $T, \bar{T}$, now coupling to a bifundamental $\Sigma$ of an enlarged $\mathrm{SU}(F)_{L} \times \mathrm{SU}(F)_{R} \times \mathrm{U}(1)_{T}$ global symmetry,

$$
W=\lambda \bar{T} \Sigma T .
$$

The Standard Model is embedded in the vector subgroup $\mathrm{SU}(F)_{V}$, and we assume the UV dynamics induces a VEV for $\Sigma,\langle\Sigma\rangle=f \times \mathbf{1}$, spontaneously breaking the global symmetry to $\mathrm{SU}(F)_{V} \times \mathrm{U}(1)_{T}$. The surviving IR degrees of freedom are the Goldstone fields $\Pi$ associated with the broken generators, parametrized as

$$
\Sigma=(f+\sigma) e^{\Pi / f} .
$$

The axial currents associated with the Goldstones $\Pi$ have a mixed $\mathrm{SU}(F)_{A}-\mathrm{SU}(F)_{V}-\mathrm{U}(1)_{T}$ anomaly, which is realized in the IR by a (supersymmetric) WZW term,

$$
W_{\mathrm{WZW}} \supset \frac{g}{16 \pi^{2} f} W_{T}^{\alpha} W_{\alpha}^{i} \Pi^{i} .
$$

This is precisely the classic supersoft operator, with the $D$-term of $W_{T}^{\alpha}$ identified as the SUSY breaking spurion.

The absence of the lemon-twist operator in the low energy theory can be demonstrated in several ways, which we do in the Appendix. It can be shown diagrammatically, which we do in Appendix A, by keeping track of the contributions to the scalar couplings from up to $\mathcal{O}\left(\Pi^{2}\right)$ terms in the UV superpotential. Alternatively, one can argue that the lemontwist operator cannot be generated because the Goldstones $\Pi$, when treated as background fields, do not contribute to the messenger masses, and hence cannot correct the $\mathrm{U}(1)_{T}$ holomorphic gauge coupling below the messenger threshold. We show this explicitly in Appendix B. Finally, one can demonstrate the absence of lemon twist in the linear realization of Goldstone gauginos, where a tadpole-induced shift in the VEV of the trace component of $\Sigma$ cancels the $B \mu$ terms that lead to tachyonic scalars, which we describe in Appendix C.

\section{A STRONGLY COUPLED MODEL}

\section{A. Warm-up: Nonrenormalizable toy model}

A crucial aspect of the GoGa mechanism that was not addressed in the previous discussion is the dynamics that induces the spontaneous breaking of the global symmetry. This dynamics can be parametrized in the simple toy model (3.1) by the inclusion of a nonrenormalizable operator such as

$$
W=\lambda \bar{T} \Sigma T+\frac{1}{\Lambda^{F-2}} S\left(\operatorname{Det} \Sigma-v^{F}\right),
$$

where $S$ is a singlet whose $F$-term induces the desired VEV for $\Sigma$.

It turns out that this symmetry breaking parametrization automatically arises in SQCD with $F=N$ flavors via the strong dynamics. In fact, all the basic ingredients for the Goldstone gaugino mechanism are naturally present in this SQCD scenario: (i) the Goldstone fields arise as composite states of the microscopic quarks, with the composite meson $M=Q \bar{Q}$ playing the analogous role of $\Sigma$ in our toy model; (ii) the UV theory has the appropriate global symmetry, namely, $\mathrm{SU}(F)_{L} \times$ $\mathrm{SU}(F)_{R} \times \mathrm{U}(1)_{B}$, (iii) which is spontaneously broken via the strong dynamics in the desired pattern due to the quantum deformation of the moduli space, and (iv) a WZW term is present in the IR to realize the mixed anomaly that sources the classic supersoft operator. In order to fully implement this strongly coupled model of Goldstone gauginos, all that is left to do is to embed the Standard Model gauge symmetries in the diagonal flavor group and induce a SUSY breaking $D$-term associated with the $U(1)_{B}$ baryon number.

This strongly coupled model provides a coherent and elegant realization of the GoGa mechanism. In the following we will review some important aspects of $F=N$ SQCD and discuss the GoGa implementation in detail. In the last subsection we will derive the classic supersoft operator starting from $F=N+1$ SQCD and integrating out the flavored baryons to recover the $F=N$ limit.

\section{B. Composite Goldstones and SUSY QCD}

A minimal realization of the Goldstone gaugino scenario can arise in models of SUSY QCD that become strongly coupled in the IR once we weakly gauge a subgroup of the theory's flavor symmetry and identify it with the SM. These theories are described in the UV by an $\mathrm{SU}(N)$ gauge group with $F$ flavors of quarks denoted by $Q$ and $\bar{Q}$. The IR degrees of freedom are weakly coupled composite mesons $M=Q \bar{Q}$, and for $F \geq N$ also baryons $B=Q^{N}$ and $\bar{B}=$ $\bar{Q}^{N}[63,64]$. The strong coupling scale is related to the lowest component of the holomorphic scale, $\Lambda^{b}$, with $b=$ $3 N-F$ the one-loop beta function for the $\mathrm{SU}(N)$ gauge 
TABLE I. Global symmetries and charges for the UV and IR degrees of freedom of SUSY QCD. The diagonal flavor subgroup $\mathrm{SU}(F)_{V}$ is gauged and identified with the SM.

\begin{tabular}{lcrrrrrr}
\hline \hline & $\mathrm{SU}(N)$ & $\mathrm{SU}(F)_{L}$ & $\mathrm{SU}(F)_{R}$ & $\mathrm{U}(1)_{B}$ & $\mathrm{U}(1)_{A}$ & $\mathrm{SU}(F)_{V}$ & $\mathrm{U}(1)_{R}$ \\
\hline$Q$ & $\square$ & $\square$ & 1 & 1 & 1 & $\square$ & $\frac{F-N}{F}$ \\
$\bar{Q}$ & $\square$ & 1 & $\square$ & -1 & 1 & $\square$ & $\frac{F-N}{F}$ \\
$M$ & 1 & $\square$ & $\square$ & 0 & 2 & $1+$ Adj. & $2 \cdot \frac{F-N}{F}$ \\
$B$ & 1 & $\left.\overline{(} \begin{array}{c}F \\
N\end{array}\right)$ & 1 & $N$ & $N$ & $\frac{(F}{(F)}$ & $N \cdot \frac{F-N}{F}$ \\
$\bar{B}$ & 1 & 1 & $\left(\begin{array}{l}F \\
N\end{array}\right)$ & $-N$ & $N$ & $\left(\begin{array}{c}F \\
N\end{array}\right)$ & $N \cdot \frac{F-N}{F}$ \\
$\Lambda^{b}$ & 1 & 1 & 1 & 0 & $2 F$ & 1 & 0 \\
\hline \hline
\end{tabular}

coupling. For reference, we list the symmetries and field content of this class of theories in Table I.

The dynamics underlying the generation of a Dirac gaugino mass is illustrated in Fig. 1. We note that the gaugino is odd under a $C$-parity that exchanges $Q$ and $\bar{Q}$ while the mesino $\psi_{M}$ is even, so the diagram must involve a breaking of this symmetry. The breaking can enter through a splitting of the squark soft masses, ${ }^{3}$ which, apart from anomaly-mediated terms, will constitute the only source of SUSY breaking on the UV variables in our setup.

Moreover, the strong dynamics inducing the $\langle Q \bar{Q}\rangle$ condensate is also responsible for $Q \leftrightarrow \bar{Q}$ exchange in the diagram, allowing the external state on the right-hand side to be identified with the mesino $\psi_{M}$.

Before proceeding, we note two main advantages we stand to realize in this setup:

(i) The desired symmetry breaking vacuum $\langle M\rangle \propto 1$ in a supersymmetric theory can be a natural consequence of the strong dynamics, rather than being enforced through dedicated model building;

(ii) The theory above the strong coupling scale can be governed by a smaller number of degrees of freedom, allowing for models where SM gauge couplings remain weak below the GUT scale and recovering the possibility of perturbative unification.

\section{Realization in $F=N$ SUSY QCD}

The Goldstone gaugino mechanism emerges quite simply in an $\mathrm{SU}(N)$ gauge theory with $F=N$ flavors of quarks $Q$, $\bar{Q}$, transforming as an $N$ and $\bar{N}$ of $\mathrm{SU}(N)$, respectively. The theory is described by a manifold of supersymmetric vacua parametrized by meson $\left(M_{j}^{i}\right)$ and baryon $(B, \bar{B})$ moduli, with certain combinations of these corresponding to physical states at low energy. The moduli space is deformed at the quantum level, according to the constraint equation

$$
\operatorname{det} M-B \bar{B}=\Lambda^{2 N},
$$

\footnotetext{
${ }^{3}$ We can express UV soft masses as projections onto $\mathrm{U}(1)_{A}$ and $\mathrm{U}(1)_{B}$ directions, such that the mass insertion in Fig. 1 corresponds to a baryon current. We expand on this below.
}

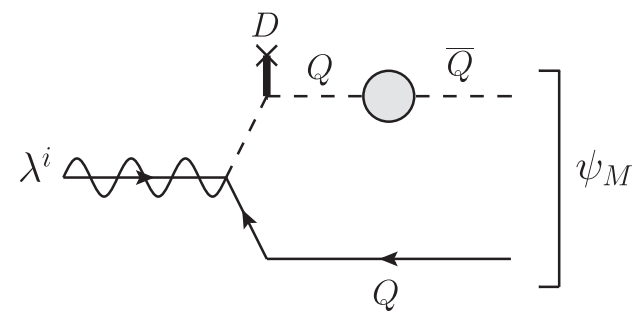

FIG. 1. Schematic constituent diagram of gaugino-mesino coupling generating the Dirac gaugino state in the IR theory. The $D$-term insertion reflects $C$-breaking soft masses for the microscopic squarks $\left(\tilde{m}_{Q}^{2} \neq \tilde{m}_{\bar{Q}}^{2}\right)$, and the blob indicates the strong dynamics underlying the $\langle Q \bar{Q}\rangle$ condensate. An analogous diagram with $Q \leftrightarrow \bar{Q}$ also contributes to the Dirac gaugino mass.

which signals the spontaneous breaking of the $\mathrm{SU}(F)_{L} \times$ $\mathrm{SU}(F)_{R} \times \mathrm{U}(1)_{B} \times \mathrm{U}(1)_{R}$ global symmetry. Thus the Goldstone superfield providing the right-handed gaugino can emerge naturally as a composite state of such a theory expanded around a vacuum $M_{j}^{i}=\Lambda^{2} \delta_{j}^{i}, B \bar{B}=0^{4}$ once the appropriate flavor symmetries are weakly gauged.

We therefore assume a vacuum where $M \propto \mathbf{1}_{N}$, in such a way that $N^{2}-1$ meson components (the traceless component of $M$ ) are identified as Goldstones. We parametrize these fields as

$$
\begin{aligned}
M & =\Lambda(\Lambda+\sigma) e^{\Pi^{i} T^{i} / f}, \\
\left.\Pi^{i}\right|_{\theta=\bar{\theta}=0} & =s^{i}+i \pi^{i}, \\
f & \sim \Lambda,
\end{aligned}
$$

with $T^{i}$ denoting the generators of $\mathrm{SU}(F)_{V}$ under which $\Pi^{i}$ transforms as an adjoint. ${ }^{5}$ The constraint (3.2) then imposes to leading order

$$
\sigma=\frac{1}{N \Lambda^{2 N-1}} B \bar{B}
$$

removing the singlet meson superfield from the theory.

The classic supersoft operator in this theory arises as a WZW term once we gauge the diagonal flavor group, $\mathrm{SU}(F)_{V}$, and identify it with the SM gauge group or one of its simple factors. Specifically the UV theory possesses an $\mathrm{SU}(F)_{A}-\mathrm{SU}(F)_{V}-\mathrm{U}(1)_{B}$ mixed anomaly ${ }^{6}$ which is not

\footnotetext{
${ }^{4}$ As we shall see, it is natural for one of the baryons to acquire a VEV of $\mathcal{O}(\Lambda)$, but since the other baryon will have a large soft mass, it is energetically favorable to satisfy the constraint (3.2) with a meson VEV.

${ }^{5}$ Because of the incalculability of the Kähler potential away from the origin of the moduli space, it is not possible to determine the numerical ratio between $f$ and $\Lambda$. A canonical Kähler potential of the form $\Delta K=\operatorname{Tr}\left(M^{\dagger} M\right) / \Lambda^{2}$ would imply $f=\Lambda$ and a canonical normalization of $\sigma$ such that $\sigma \rightarrow \sigma / \sqrt{N}$ in Eq. (3.3).

${ }^{6} \mathrm{We}$ use this to denote the combination of $\mathrm{SU}(F)_{L, R}^{2}-\mathrm{U}(1)_{B}$ anomalies containing the current to which the Goldstones couple.
} 
realized by the IR fermionic degrees of freedom. Instead the anomaly is realized by the Goldstones via a supersymmetric WZW term

$$
W_{\mathrm{WZW}} \supset \frac{g N}{16 \pi^{2} f} W_{B}^{\alpha} W_{\alpha}^{i} \Pi^{i}
$$

with field strengths $W_{B}^{\alpha}$ for $\mathrm{U}(1)_{B}$ and $W^{\alpha}$ for $\mathrm{SU}(F)_{V}$. This form of the WZW term will be derived in the following subsection starting from SQCD with $F=N+1$ flavors, where all anomalies are saturated by loops of the IR fermions. We will then generate the WZW term and recover the $F=N$ limit by integrating out the flavored baryons of the theory.

Equation (3.5) implies that the $D$-term encoding SUSY breaking in the IR spectrum can be traced to a splitting of the microscopic squark soft masses. To understand the SUSYbreaking spurion in the IR, we recall that Kähler terms $\Delta K=$ $Z Q^{\dagger} Q$ admit a background $\mathrm{U}(1)$ gauge symmetry

$$
Q \rightarrow e^{-\Omega} Q, \quad Z \rightarrow e^{\Omega+\Omega^{\dagger}} Z,
$$

with $\Omega$ a chiral superfield and $\ln Z$ transforming as the $\mathrm{U}(1)$ gauge field,

$$
\ln Z \rightarrow \ln Z+\Omega+\Omega^{\dagger}
$$

This allows the construction of an invariant "field strength" for the vector superfield $\ln Z$; cf. [65] for details. Allowing for $\tilde{m}_{Q}^{2} \neq \tilde{m}_{\bar{Q}}^{2}$ via distinct wave function factors for the fields, we thus have

$$
W_{B}^{\alpha}=-\frac{1}{4} \bar{D}^{2} D^{\alpha} \ln \left(\frac{Z_{Q}}{Z_{\bar{Q}}}\right) \supset 2 \tilde{m}_{\mathcal{V}}^{2} \theta^{\alpha},
$$

where we use the notation

$$
\tilde{m}_{\mathcal{A}}^{2} \equiv \frac{\tilde{m}_{Q}^{2}+\tilde{m}_{\bar{Q}}^{2}}{2}, \quad \tilde{m}_{\mathcal{V}}^{2} \equiv \frac{\tilde{m}_{Q}^{2}-\tilde{m}_{\bar{Q}}^{2}}{2}
$$

for soft masses projected along the axial and vector $\mathrm{U}(1)$ directions, respectively. We thereby recover the Dirac gaugino mass generated by the classic supersoft operator (3.5) as

$$
m_{D}=\frac{g N}{16 \pi^{2}} \frac{\tilde{m}_{\mathcal{V}}^{2}}{f} .
$$

Soft masses for the microscopic SQCD squarks are expected also to feed into the real scalar components of the Goldstone superfields. Assuming a lowest order Kähler term for the mesons of the form

$$
\Delta K \propto \frac{Z_{Q} Z_{\bar{Q}}}{\Lambda_{I}^{2}} \operatorname{Tr}\left(M^{\dagger} M\right)
$$

with normalization related to the invariant scale $\Lambda_{I}^{2}=$ $\Lambda^{\dagger} Z_{Q}^{F / b} Z_{\bar{Q}}^{F / b} \Lambda$ of [66], and then expanding in powers of $\theta$ reveals soft mass terms for the scalars, $s$,

$$
\tilde{m}_{s}^{2} \propto \tilde{m}_{\mathcal{A}}^{2}
$$

The pseudoscalars, on the other hand, are left massless by (3.11); this is exactly as required by simple symmetry arguments, since the SQCD squark soft masses do not break the global symmetry, meaning that the masses of the $\pi$ fields remain protected by Goldstone's theorem.

One potential issue that needs to be addressed is the generation of a tachynic soft mass for one of the baryons in the presence of a $U(1)_{B} D$-term. The easiest way to see this is to introduce a $\mathrm{U}(1)_{B}$ Fayet-Iliopoulos term, and to gauge $\mathrm{U}(1)_{B}$ so that the auxiliary field $D$ couples to the scalar baryons in the Kähler potential in the usual way. ${ }^{7}$ After integrating out the auxiliary field $D$, a tachyonic mass is induced for one of the baryons. This need not be a problem, however, since the SQCD baryons do not carry SM charges. In this case, we expect a Kähler potential (neglecting interactions with other fields) containing a general polynomial in $B$ and $B^{*}$, with the leading term being $-\tilde{m}_{\nu}^{2} B B^{*}$ and with higher terms suppressed by the scale $\Lambda$. For this scenario to be viable, this baryonic direction cannot be a runaway, but instead the polynomial must have the (very reasonable) property that it has a minimum somewhere with characteristic value $\Lambda$. Alternatively, these baryons could acquire mass from a higher dimension operator $\mu^{4} Q^{3} \bar{Q}^{3} / M_{*}^{3}$, with $M_{*}>\Lambda$. For this to work, however, both $M_{*}$ and $\Lambda$ should be very high scales to allow the mass $\mu^{4} / M_{*}^{3}$ to be larger than $\tilde{m}_{\mathcal{V}}$ and compensate for the tachyonic soft mass. Regardless, this tachyonic direction is a far less serious concern in that it does not apply to any of the fields which must be present in the low energy theory, and a baryon VEV does not cause any fundamental problems as long as it does not run away.

Last, we comment on the matching of additional anomalies between the UV and IR theories. In ordinary SQCD with ungauged flavored symmetries, there is, for example, a $U(1)_{R} \mathrm{SU}(F)^{2}$ anomaly matched in the IR by the mesino $\psi_{M}$. One might naively worry that this anomaly is not matched in the deep IR once the mesino marries the SM gaugino and is integrated out. That is not the case, however. Different from SQCD with ungauged flavor symmetries, in our scenario the gauging of some flavor symmetries introduces extra fermionic degrees of freedom to the theory, namely the SM gauginos. These extra fermions contribute so as to cancel the $U(1)_{R} \mathrm{SU}(F)^{2}$ anomaly, hence preserving the t'Hooft anomaly matching conditions. The same reasoning applies to all the other anomalies realized by the composite fermions in our scenario with gauged flavor symmetries.

\footnotetext{
${ }^{7}$ Additionally the baryon mass matrix receives an off-diagonal component due to a linear term generated for the singlet (trace) component of the meson.
} 


\section{Connecting with $F \neq N$}

The structure of the low energy theory with $F=N$ can be illuminated by starting in a theory with $F=N+1$ flavors - whose details we briefly recap - and adding a supersymmetric mass for one flavor.

In SQCD with $F=N+1$, the origin remains on the quantum moduli space (that is, the theory $s$-confines), and all of the mesons and baryons correspond to light physical states. We will denote these as $\mathcal{M}$ and $\mathcal{B}, \overline{\mathcal{B}}$, respectively. Deforming the theory such that $\mathcal{M}$ moves away from the origin, the baryons decouple via the theory's superpotential $W=\mathcal{B} \mathcal{M} \overline{\mathcal{B}}$ and any anomalies matched by these baryons must translate into WZW terms in the surviving $F=N$ theory. This nondecoupling effect of the baryons is clearly seen by performing a standard perturbative loop calculation and mirrors the role of "constituent quarks" in ordinary QCD [67].

In the $F=N+1$ theory the meson directions develop VEVs proportional to supersymmetric $Q \bar{Q}$ mass terms. With $\Lambda_{N+1}$ indicating the strong coupling scale in this theory, the vacuum is described by

$$
\left\langle\left(\mathcal{M}^{-1}\right)_{j}^{i} \operatorname{det} \mathcal{M}\right\rangle=m_{j}^{i} \Lambda_{N+1}^{2 N-1},
$$

indicating that fluctuations of the composites do not lie on the moduli constraint surface. Classical relationships between the composites therefore arise as equations of motion, which can be appropriately arranged with

$$
W=\frac{1}{\Lambda_{N+1}^{2 N-1}}(\mathcal{B} M \overline{\mathcal{B}}-\operatorname{det} \mathcal{M}) \text {. }
$$

Baryons thus become massive as $\langle\mathcal{M}\rangle \neq 0$.

To recover the $F=N$ case, we introduce a mass for the $F$ th flavor,

$$
\Delta W=m Q^{F} \bar{Q}_{F}=m \mathcal{M}_{F}^{F},
$$

and decompose the composite fields as

$$
\mathcal{M}=\left(\begin{array}{cc}
M & X \\
\bar{X} & \mathcal{M}_{F}^{F}
\end{array}\right), \quad \mathcal{B}=\left(\begin{array}{c}
Y \\
B
\end{array}\right)^{T}, \quad \overline{\mathcal{B}}=\left(\begin{array}{c}
\bar{Y} \\
\bar{B}
\end{array}\right),
$$

where $M, X, \bar{X}, Y$, and $\bar{Y}$ transform under the $\square \times \bar{\square}$, $\mathbf{1} \times \bar{\square}, \square \times \mathbf{1}, \bar{\square} \times \mathbf{1}$, and $\mathbf{1} \times \square$ representations of $\mathrm{SU}(F-1)_{L} \times \mathrm{SU}(F-1)_{R}$, respectively, and $\mathcal{M}_{F}^{F}, B$, and $\bar{B}$ are singlets. The vacuum condition (3.13) becomes

$$
\operatorname{det} M-B \bar{B}=m \Lambda_{N+1}^{2 N-1}=\Lambda_{N}^{2 N},
$$

where the usual scale matching between the $F=N+1$ and $F=N$ theories provides the second equality. The $Y, \bar{Y}$ and $X, \bar{X}$ fields become massive and can be integrated out. We shall now discuss the effects of integrating out $Y, \bar{Y}$.

The $Y, \bar{Y}$ baryons mediate mixed $\mathrm{SU}(F)^{2} \mathrm{U}(1)_{B}$ anomalies, and once integrated out (taking $m \rightarrow \infty$ ), the $F=N$ limit is recovered and WZW terms must arise to realize those anomalies. Indeed we will check appropriate diagrams explicitly. Using the parametrization Eq. (3.3) for $M$, the pertinent superpotential couplings are

$$
\Delta W=m Y e^{\Pi / f} \bar{Y},
$$

where $Y, \bar{Y}$ have been normalized to have canonical dimension, i.e. $Y / \Lambda_{N}^{N-1} \rightarrow Y$ and $\bar{Y} / \Lambda_{N}^{N-1} \rightarrow \bar{Y}$ in Eq. (3.14). At linear order we obtain the following interactions for the scalar components of $\Pi$ :

$$
\Delta \mathcal{L}=-m\left(1+\frac{s^{a}+i \pi^{a}}{f} T^{a}\right) \psi \psi^{c}+\text { H.c. },
$$

where $\psi, \psi^{c}$ are the (left-handed Weyl) fermion components of $Y$ and $\bar{Y}$. We can now proceed with familiar component calculations to recover the various pieces of the $F=N$ theory's WZW term, Eq. (3.5).

Consider the pseudoscalar $\pi$ for example. From a threepoint diagram involving $\pi$ and two gauge bosons we extract the $\mathcal{O}\left(p^{2}\right)$ piece, which is the only term that survives as $m \rightarrow \infty$; one fermion propagator therefore contributes proportional to its mass rather than momentum. Hence the nondecoupling effect of $Y, \bar{Y}$ on the $\mathrm{SU}(F)_{A}-\mathrm{SU}(F)_{V}-\mathrm{U}(1)_{B}$ anomaly gives

$$
\begin{aligned}
= & =\frac{N}{4 \pi^{2} f} \delta^{a b} \epsilon^{\mu \nu \alpha \beta} p_{\alpha}^{(1)} p_{\beta}^{(2)} .
\end{aligned}
$$

Here the cross indicates the mass insertion $m ; p^{(1,2)}$ are the momenta entering the gauge vertices, and the factor of $N$ comes from the $\mathrm{U}(1)_{B}$ charge of the fermions. ${ }^{8}$ This result implies a WZW term $[68,69]$ in the low-energy Lagrangian,

\footnotetext{
${ }^{8}$ We have taken the baryon number to be gauged in this component calculation. We may ultimately turn its gauge coupling off since the $\mathrm{U}(1)_{B}$ field strength needs to act only through its $\mathcal{O}(\theta)$ SUSY breaking spurion.
} 


$$
\Delta \mathcal{L}=-\frac{g N}{16 \pi^{2} f} \pi^{i} F_{\mu \nu}^{i} \tilde{B}^{\mu \nu}
$$

This particular derivation parallels that of the $\pi^{0} \rightarrow \gamma \gamma$ computation with constituent quarks in QCD [67].

The WZW term (3.21) can be written as part of a superpotential, and we can confirm with similar manipulations to those above that terms related to it by SUSY are consistently reproduced by taking

$$
\Delta W=\frac{g N}{16 \pi^{2} f} W_{B}^{\alpha} W_{\alpha}^{i} \Pi^{i},
$$

and hence we have recovered the classic supersoft operator (3.5).

\section{A PERTURBATIVE MODEL}

Even though strongly-coupled models offer a natural UV completion for Goldstone gauginos, perturbative and renormalizable UV completions are also possible. In this section we discuss one such possibility. The basic ingredients discussed in Sec. II are easily incorporated, such as a messenger sector coupling to bifundamentals of an approximate chiral symmetry containing the SM as a subgroup. To ensure the generation of the classic supersoft operator, the spontaneous breaking of the global symmetry must be arranged such that the currents associated with the Goldstone fields are anomalous with respect to the SM and the U(1) carrying the SUSY breaking $D$-term.

In the model we will discuss, a larger messenger sector will be necessary to avoid destabilizing the symmetry breaking vacuum. The model will consist simply of two replicas of the toy model discussed in Sec. II,

$$
W_{\text {mess }}=\lambda \bar{T}_{1} \Sigma_{1} T_{1}+\lambda T_{2} \Sigma_{2} \bar{T}_{2},
$$

where the equality of couplings is enforced by an exchange symmetry $1 \leftrightarrow 2$. We will discuss the symmetry breaking sector shortly. This messenger sector by itself is invariant under an enlarged global symmetry, $\mathrm{SU}(F)_{L 1} \times \mathrm{SU}(F)_{R 1} \times$ $\mathrm{U}(1)_{T 1} \times \mathrm{U}(1)_{A 1}$ and $\mathrm{SU}(F)_{L 2} \times \mathrm{SU}(F)_{R 2} \times \mathrm{U}(1)_{T 2} \times$ $\mathrm{U}(1)_{A 2}$, acting independently on each of the two replicas. The $A$ subscript denotes an axial symmetry. Supposing for the moment that both $\Sigma_{1}$ and $\Sigma_{2}$ would obtain diagonal VEVs, $\left\langle\Sigma_{1}\right\rangle=\left\langle\Sigma_{2}\right\rangle=f \times \mathbf{1}$, the global symmetry would be broken to the diagonal subgroups, $\mathrm{SU}(F)_{V 1} \times \mathrm{U}(1)_{T 1}$ and $\mathrm{SU}(F)_{V 2} \times \mathrm{U}(1)_{T 2}$, and the low energy theory would have $2 F^{2}$ Goldstones, $\Pi_{1}$ and $\Pi_{2}$, such that

$$
\begin{aligned}
\Sigma_{1,2} & =\left(f+\sigma_{1,2}\right) e^{\Pi_{1,2} / f}, \\
\left.\Pi_{1,2}\right|_{\theta, \bar{\theta}=0} & =s_{1,2}+i \pi_{1,2} .
\end{aligned}
$$

Moreover, there would be two independent mixed anomalies as displayed below.
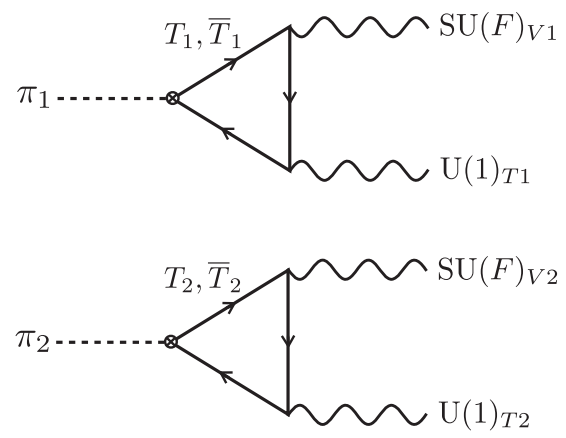

The symmetry breaking sector, however, will not respect the full chiral symmetry of the messenger sector, but only the chiral subgroup $\mathrm{SU}(F)_{L} \times \mathrm{SU}(F)_{R} \times \mathrm{U}(1)_{A}$ where $L=L_{1}+L_{2}$ and $R=R_{1}+R_{2}$, under which $\Sigma_{1}$ and $\Sigma_{2}$ transform as $(\square, \bar{\square}, 1)$ and $(\bar{\square} \square,-1)$, respectively. In reality, therefore, there will only be $F^{2}$ Goldstone bosons resulting from the spontaneous breaking $\mathrm{SU}(F)_{L} \times$ $\mathrm{SU}(F)_{R} \times \mathrm{U}(1)_{A} \rightarrow \mathrm{SU}(F)_{V} \times \mathrm{U}(1)_{A}$, with $F^{2}-1$ of them given by the linear combination $\pi=\pi_{1}-\pi_{2}$. The mixed anomaly sourcing the classic supersoft operator will be $\mathrm{SU}(F)_{A}-\mathrm{SU}(F)_{V}-\mathrm{U}(1)_{T},{ }^{9}$ schematically displayed below (where $T=T_{1}-T_{2}$ denotes the messenger number).

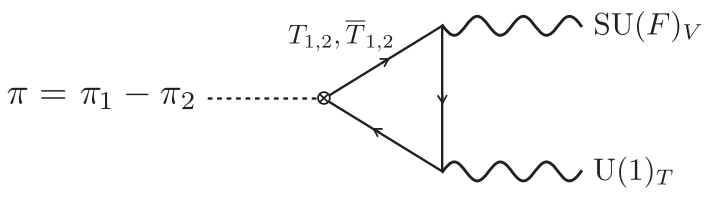

This mixed anomaly is manifested in the IR by a WZW term,

$$
W_{\mathrm{WZW}} \supset \frac{g}{16 \pi^{2} f} W_{T}^{\alpha} W_{\alpha}^{i} \Pi^{i},
$$

where $\Pi^{i}=\Pi_{1}^{i}-\Pi_{2}^{i}$. As before, we recognize this as the classic supersoft operator, realizing the Goldstone gaugino mechanism once it is arranged for $\left\langle W_{T}^{\alpha}\right\rangle=D \theta^{\alpha}$.

We can now finally introduce the spontaneous symmetry breaking sector,

$$
W_{\text {SSB }}=\kappa_{1} S\left(\operatorname{Tr}\left[\Sigma_{1} \Sigma_{2}\right]-v^{2}\right)+\kappa_{2} \operatorname{Tr}\left[\Sigma_{1} \Omega \Sigma_{2}\right] \text {, }
$$

where, as before, $\Sigma_{1}$ and $\Sigma_{2}$ are in the $(\square, \bar{\square}, 1)$ and $(\bar{\square}, \square,-1)$ representations of $\mathrm{SU}(F)_{L} \times \mathrm{SU}(F)_{R} \times \mathrm{U}(1)_{A}$, respectively, and we have introduced two new chiral superfields $S$ and $\Omega$ transforming as $(\mathbf{1}, \mathbf{1}, 0)$ and $(\mathbf{1}$, Ad, 0$)$, respectively.

The first term in (4.4) induces a VEV to the trace components of $\Sigma_{1}, \Sigma_{2}$, such that $\left\langle\Sigma_{1}\right\rangle=\alpha v / \sqrt{F} \times \mathbf{1}$ and

\footnotetext{
${ }^{9}$ Because of the doubling of messengers there are in principle two different $\mathrm{U}(1)_{T}$ symmetries. We allow a nonzero $D$-term for the combination under which the charge of $T_{1}$ is opposite to the charge of $T_{2}$.
} 
$\left\langle\Sigma_{2}\right\rangle=\alpha^{-1} v / \sqrt{F} \times \mathbf{1}$. The ratio of these VEVs, parametrized by $\alpha$, need not be one; it represents a flat direction of the potential that in principle will be lifted by SUSY breaking. If $\alpha \neq 1$, the low energy Goldstones would be given by a different admixture of $\Pi_{1}, \Pi_{2}$, but otherwise our previous discussion of the low energy theory remains unchanged.

Finally, the second term in the superpotential (4.4) is present to give masses to the remaining degrees of freedom upon spontaneous breaking of the global symmetry. The components of $\Omega$ pair up with the combination of $\Pi_{1}$ and $\Pi_{2}$ orthogonal to the Goldstone $\Pi$, and the trace components of $\Sigma_{1}$ and $\Sigma_{2}$ orthogonal to the axial Goldstone pair up with the singlet $S$ to obtain a Dirac mass. Thus there are $\left(F^{2}-1\right)$ Goldstones in the adjoint of the unbroken $\mathrm{SU}(F)_{V}$ symmetry, which may be identified with a SM gauge group, and a singlet Goldstone associated with the spontaneous breaking of the $\mathrm{U}(1)_{A}$ axial symmetry. Coupling of either $\Sigma_{1}$ or $\Sigma_{2}$ to a pair of messenger fields leads to a tadpole term for this singlet which picks a vacuum $\alpha \neq 1$; however, equal coupling of each field to its own pair of messengers in Eq. (4.1) avoids this issue as each tadpole term cancels and the calculation is consistent with remaining at the minimum of the scalar potential.

We have shown with this example that it is possible to construct simple renormalizable and perturbative models of Goldstone gauginos. In general, all the arguments we have presented carry forward when we parametrize the Goldstone fields as exponentials. At the same time, we should be able to see the absence of the lemon twist by studying the linearized model and including all of the heavy fields as well. In Appendix C, we revisit the nonrenormalizable toy model in (3.1) in the linear representation of the Goldstone fields; we demonstrate that lemon twist is not generated due to a cancellation of scalar soft masses by a tadpole-induced shift in the trace component of $\Sigma$.

\section{IMPLICATIONS AND DISCUSSION}

\section{A. Global symmetry breaking operators}

The Goldstone gaugino mechanism depends crucially on the approximate global symmetry of the UV theory to protect it from dangerous tachyonic masses. However, there are explicit breaking sources of the global chiral symmetry $\mathrm{SU}(F)_{L} \times \mathrm{SU}(F)_{R}$, such as the SM gauging of the vector subgroup $\mathrm{SU}(F)_{V}$, or (potentially) nonperturbative gravitational effects. Those could induce chiral symmetry breaking operators in the Kähler potential or superpotential, which may source large Majorana masses for the righthanded gaugino and/or large masses for the adjoint (pseudo)scalars, thus spoiling the GoGa mechanism. In the following we will discuss the effects these breaking sources and the necessary conditions to ensure that the GoGa mechanism is preserved. We will use the notation of the toy model described at the end of Sec. II.

\section{Gravitational breaking}

The first chiral symmetry breaking operators we will consider are those generated by nonperturbative gravitational effects. ${ }^{10}$ In particular, they could be independent of the SUSY breaking spurion $W_{T}^{\alpha}$ or any explicit breaking parameters (such as SM gauge couplings). An example of such operator in the superpotential would be

$$
\Delta W=\frac{1}{M_{\mathrm{Pl}}^{n-3}} \operatorname{Tr}\left[\Sigma^{n}\right]
$$

which would induce a holomorphic mass to the Goldstone chiral adjoint $\Pi$ scaling as

$$
M_{\Pi} \propto \frac{f^{n-2}}{M_{\mathrm{Pl}}^{n-3}} .
$$

For $n<4$ the GoGa mechanism would be spoiled; that, however, can easily be avoided by gauging a discrete $Z_{4}$ symmetry which acts on $\Sigma$ and forbids unsuppressed holomorphic adjoint masses. If the $n=4$ operator is present, the induced Majorana mass for the right-handed gaugino will be small as long as $f \ll \sqrt{m_{D} M_{\mathrm{Pl}}}$.

Additionally, gravity could also induce chiral symmetry breaking operators in the Kähler potential, such as

$$
\Delta K=\frac{1}{M_{\mathrm{Pl}}^{2 p-2}} \operatorname{Tr}\left[\Sigma^{\dagger p}\right] \operatorname{Tr}\left[\Sigma^{p}\right]
$$

which cannot not be forbidden by discrete symmetries. The operator with $p=1$ in particular is unsuppressed; however, being in the Kähler potential, it does not generate supersymmetric masses for the adjoint fields. Its effect will be to renormalize holomorphic masses such as (5.2) and, in the presence of SUSY breaking, induce masses for the pseudoscalar adjoints at one-loop scaling as

$$
\delta m_{\pi}^{2} \propto \frac{m_{D}^{4}}{f^{2}} .
$$

Assuming, however, a mild hierarchy between $f$ and $m_{D}$ is sufficient to guarantee that (5.4) is small and that the GoGa mechanism is preserved.

It is worth noting that the strongly coupled model is further protected from the effects discussed above. For instance, operators of the type (5.1) would stem from

$$
\Delta W=\frac{1}{M_{\mathrm{Pl}}^{2 n-3}}(Q \bar{Q})^{n} \rightarrow \frac{\Lambda^{n}}{M_{\mathrm{Pl}}^{2 n-3}} \operatorname{Tr}\left[M^{n}\right],
$$

and the corresponding holomorphic adjoint mass would instead scale as

\footnotetext{
${ }^{10}$ For the ease of notation, we will omit the coefficients of gravity-induced operators, which we will implicitly assume are $\mathcal{O}(1)$.
} 


$$
M_{\Pi} \propto \frac{\Lambda^{2 n-2}}{M_{\mathrm{Pl}}^{2 n-3}},
$$

which is safe as long as $n>1$ and $\Lambda \ll\left(m_{D} M_{\mathrm{Pl}}^{2 n-3}\right)^{1 /(2 n-2)}$. Moreover, (5.3) would stem from

$\Delta K=\frac{1}{M_{\mathrm{Pl}}^{4 p-2}}\left(Q^{\dagger} \bar{Q}^{\dagger}\right)^{p}(Q \bar{Q})^{p} \rightarrow \frac{\Lambda^{2 p}}{M_{\mathrm{Pl}}^{4 p-2}} \operatorname{Tr}\left[M^{\dagger p}\right] \operatorname{Tr}\left[M^{p}\right]$,

which is safe even for $p=1$ since it contributes to the pseudoscalar adjoint mass as

$$
\delta m_{\pi}^{2} \propto \frac{m_{D}^{4}}{M_{\mathrm{Pl}}^{2}} .
$$

Finally, we consider operators that depend on the SUSY breaking spurion, namely $W_{T}^{\alpha} \equiv D \theta^{\alpha}$ or $V_{T} \equiv D \theta^{2} \bar{\theta}^{2}$. If the $\mathrm{U}(1)_{T}$ symmetry associated with such spurion is a global symmetry, then gravity could generate operators in the Kähler potential such as

$$
\Delta K=V_{T} \operatorname{Tr}\left[\Sigma^{\dagger}\right] \operatorname{Tr}[\Sigma] .
$$

Depending on the sign of (5.9), either the scalar or the pseudoscalar adjoint will get a large tachyonic mass scaling as $\delta m^{2} \propto-f m_{D}$ [in the strongly coupled scenario there is an additional Planck suppression $\left.\delta m^{2} \propto-\left(\Lambda / M_{\mathrm{Pl}}\right)^{2} \Lambda m_{D}\right]$. If instead $\mathrm{U}(1)_{T}$ were an actual gauge symmetry, (5.9) could still be generated, but with a suppression of $\left(v_{T} / M_{\mathrm{Pl}}\right)^{2}$ [where $v_{T}$ is the VEV that spontaneously breaks $\mathrm{U}(1)_{T}$ ]. Assuming $v_{T} \ll M_{\mathrm{Pl}}$ is sufficient to protect the GoGa mechanism from this effect.

Further operators are either sufficiently suppressed by the Planck scale or forbidden by $\mathrm{U}(1)_{T}$ or discrete gauge symmetries.

\section{SUSY breaking (independent of SM gauge couplings)}

As we have previously discussed, LTS-type operators such as

$$
\Delta W_{\mathrm{LT}}=\frac{1}{\Lambda_{\mathrm{LT}}^{n}} W_{T}^{\alpha} W_{T \alpha} \operatorname{Tr}\left[\Sigma^{n}\right]
$$

violate the chiral and discrete gauge symmetries, and so cannot be dynamically generated.

On the other hand, higher-dimensional operators that respect those symmetries are not in principle forbidden, e.g.,

$$
\Delta K= \pm \frac{v_{T}^{2}}{\Lambda_{T}^{2}} V_{T} \operatorname{Tr}\left[\Sigma^{\dagger} \Sigma\right]
$$

Above the dependence on the SM gauge fields does not play a role and is therefore omitted. Once SUSY is broken with $\left\langle V_{T}\right\rangle=D \theta^{2} \bar{\theta}^{2}$, (5.11) will induce a large and possibly tachyonic mass for the scalar adjoint,

$$
\delta m_{s}^{2}= \pm \frac{v_{T}^{2}}{\Lambda_{T}^{2}} \frac{f}{m_{D}} m_{D}^{2},
$$

which will dominate over the contribution from the CS operator if $v_{T}>2 \Lambda_{T} \sqrt{m_{D} / f}$. In particular, in the strongly coupled model the strong dynamics might generate

$$
\Delta K=\frac{c_{B}}{\Lambda^{2}} B^{\dagger} e^{-V_{B}} B \operatorname{Tr}\left[M^{\dagger} M\right] .
$$

In principle one cannot discard the possibility that $c_{B}$ is positive and $\mathcal{O}(1)$, and $\langle B\rangle \sim \mathcal{O}(\Lambda)$, in which case the tachyon problem would reemerge. Presently, however, it is not known how to determine the Kähler potential in such theories, so we assume that operators such as (5.11) and (5.13) are sufficiently suppressed and the GoGa mechanism remains viable.

\section{SM gauging of diagonal flavor symmetry}

Next, we consider the SM gauging of the diagonal flavor symmetry, encoded in the kinetic term for $\Sigma$,

$$
K \supset \operatorname{Tr}\left[\Sigma^{\dagger} e^{-V_{\mathrm{SM}} \Sigma e^{V_{\mathrm{SM}}}}\right] .
$$

In spite of this being an explicit breaking of the chiral symmetry, it alone is not sufficient to break the shift symmetry of the pseudoscalar adjoint. That is because the adjoint masses are also protected by SUSY. More generally, as long as SUSY is preserved, any arbitrary violation of the chiral symmetry in the Kähler potential will not generate masses for the adjoint fields.

After SUSY breaking, however, adjoint masses get generated at one-loop due to (5.14). Explicit computation gives

$$
\delta m_{\pi}^{2}=\frac{\alpha_{\mathrm{SM}} C_{2}(\mathbf{A d})}{\pi} m_{D}^{2} \log \left(\frac{m_{s}^{2}}{m_{D}^{2}}\right)
$$

for the pseudoscalar adjoint, where $\alpha_{\mathrm{SM}} \equiv g_{\mathrm{SM}}^{2} /(4 \pi)$ and $C_{2}(\mathbf{A d})=N$ is the quadratic Casimir for the adjoint representation. A correction of the same order will be generated for the scalar adjoint, which is subdominant relative to the tree-level contribution $m_{s}^{2}=4 m_{D}^{2}$.

The leading $\mathcal{O}\left(\alpha_{\mathrm{SM}} m_{D}^{2}\right)$ contribution to the adjoint mass squared (5.15) shares the same origin as the supersoft masses of the Standard Model sfermions, and cannot be encoded as a local, SUSY covariant operator [14]. This contribution is universal to all Dirac gaugino scenarios (not only GoGa's), and since it is parametrically smaller than the Dirac gaugino mass, it does not spoil the GoGa mechanism. 


\section{B. Fine-tuning and naturalness}

With the tachyon problem removed, we are able to ask the question of the naturalness of the theory. While elaborate studies of fine-tuning of the electroweak scale are beyond our scope, a brief discussion of naturalness in terms of radiative corrections to the Higgs mass is warranted.

In these theories, heavy stops are insufficient to explain the Higgs mass due to the dramatic reduction in the $D$-term quartics [14], and so something along the lines of the nextto-minimal supersymmetric standard model (NMSSM) must be invoked. On the other hand, the presence of additional matter allows for larger values of the NMSSM quartic that remain perturbative in the UV [70,71], so there is a natural synergy within the setup. Even absent $D$-term quartics, we estimate that for an NMSSM strongly coupled at the grand unified theory (GUT) scale, we can have a Higgs at $125 \mathrm{GeV}$ for $1.5 \lesssim$ $\tan \beta \lesssim 2$ with sub-TeV stops.

The Higgs receives radiative corrections from the winos and binos at one-loop, and from the gluinos at two loops (via the usual stop coupling). These are [14]

$$
\begin{gathered}
\left.\delta \tilde{m}_{h}^{2}\right|_{1-\text { loop }}=\frac{2 \alpha_{2} \ln (4)}{3 \pi} m_{D 2}^{2}, \\
\left.\delta \tilde{m}_{h}^{2}\right|_{\text {2-loop }}=\frac{3}{8 \pi^{2}} y_{t}^{2} m_{\tilde{t}}^{2} \ln \left(m_{D 3} / \tilde{m}_{\tilde{t}}\right), \\
=\frac{\alpha_{3} y_{t}^{2} \ln (4)}{2 \pi^{3}} \ln \left(3 \pi / 4 \alpha_{3} \ln (4)\right) m_{D 3}^{2}, \\
\tilde{m}_{\tilde{t}}^{2} \simeq \tilde{m}_{\tilde{q}}^{2}=\frac{4 \alpha_{3} \ln (4)}{3 \pi} m_{D 3}^{2} .
\end{gathered}
$$

Here $m_{D 2}$ and $m_{D 3}$ are the masses of the wino and gluino, respectively, and we neglect the contribution from the bino.

We can make a rough assessment of the tuning of the theory by evaluating the ratio of these extra soft mass contributions to the Higgs potential relative with the observed value $m_{h}^{2}=(125 \mathrm{GeV})^{2}$. That is, we define the tuning to be $\delta \tilde{m}_{h}^{2} /\left(m_{h}^{2} / 2\right)$, and we display tuning contours in Fig. 2 as a function of gaugino and scalar masses.

We see that for a gluino mass in the $2-3 \mathrm{TeV}$ range, the tuning from the gluino contribution is $30 \%-15 \%$, with squarks in the $450-700 \mathrm{GeV}$ range. ${ }^{11}$ This marks a significant improvement over the MSSM with a comparable gluino mass.

\section{UV physics and unification}

Perturbative unification remains a challenge for Dirac gaugino models. A full $\mathbf{2 4}$ of SU(5) contains enough matter that the gauge couplings hit a Landau pole before the GUT

\footnotetext{
${ }^{11}$ Note that in the MSSM such masses would be at strong tension with existing searches. However, with heavy Dirac gauginos, "doubly invisible" decay topologies for squarks can be naturally realized [72], under which these masses are viable.
}

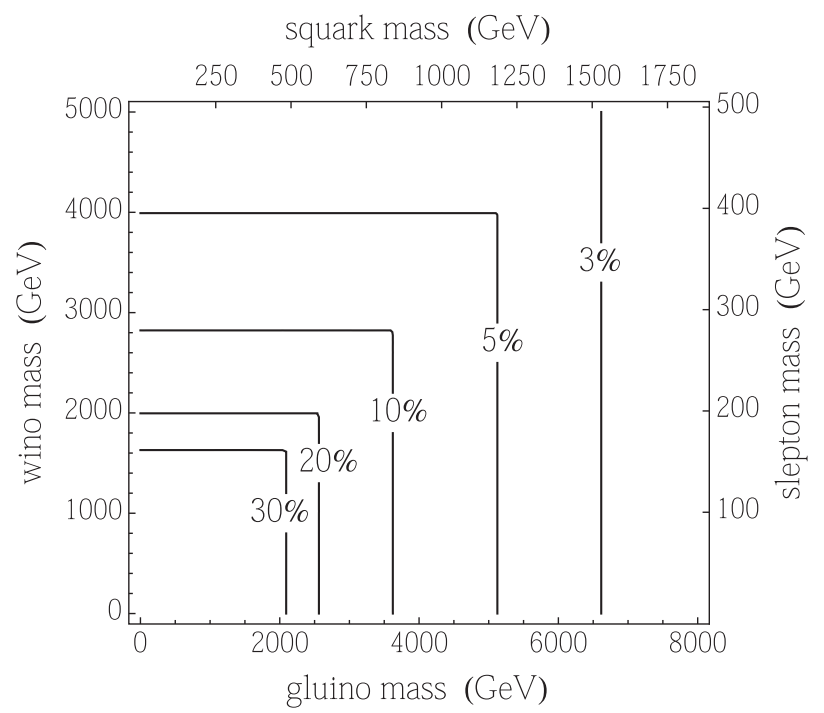

FIG. 2. Contours of electroweak scale tuning (shown as percentages), determined by the ratio of the largest radiative correction of the Higgs mass to its observed value of $(125 \mathrm{GeV})^{2}$. Radiative contributions to squark and slepton masses from the associated gaugino mass are also shown.

scale $[73,74]$. In the context of composite models of Goldstone gauginos, we have interesting possibilities, however. It is possible that the low energy theory contains SM adjoints built out of confining fundamentals. For instance, one can consider a gauge group $\mathrm{SU}(3)_{a} \times$ $\mathrm{SU}(3)_{b} \times \mathrm{SU}(3)_{\mathrm{SM}} \times \mathrm{SU}(2)_{\mathrm{SM}} \times U(1)_{\mathrm{SM}}$, with an $a \leftrightarrow b$ symmetry of couplings imposed at the GUT scale. With fields $d_{a}=(3,1,3,1,1 / 3)$ and $\ell_{b}=(1,3,1,2,1 / 2)$ (and vectorlike partners), the SM $\beta$ functions are shifted by the equivalent of three flavors. Such a model could arise out of an orbifold GUT, for instance.

When $\mathrm{SU}(3)_{a}$ and $\mathrm{SU}(3)_{b}$ confine, the theory produces composite adjoints of the SM gauge group, and in addition two sets of baryons with hypercharges \pm 1 , and doublets with hypercharge $\pm 1 / 2$. Together, this maintains a unified shift to the SM $\beta$-functions. Indeed, this is quite analogous to the (charged) matter present in the adjoint in trinification $[75,76]$, but the high energy theory is arising from a set of split SU(5) multiplets. It is difficult to employ this model, however, as the tachyonic baryons would have hypercharge. This could be solved with explicit masses for the microscopic quarks of the strongly coupled sector, but then supersoftness would be retained only if $\Lambda \sim m_{\text {SUSY }}$.

As a first alternative, we can look to models where the fields $d_{a}$ carry no hypercharge; these models do not generically unify, so we will discuss a unifying case subsequently. Considering the $\mathrm{SU}(3)_{b}$ gauge group to be associated with its own $\mathrm{SU}(3)_{b L} \times \mathrm{SU}(3)_{b R}$ chiral symmetry, the idea is to identify hypercharge with the diagonal subgroup's Abelian generator $\lambda^{8} \propto \operatorname{diag}(1,1,-2)$; tracelessness then ensures that all baryons will be completely neutral under the SM gauge group. Specifically, let us take 


$$
Y=\sqrt{3} c \lambda^{8}=\operatorname{diag}(c / 2, c / 2,-c) .
$$

Relative to the wino, the bino's mass is thus scaled by an amount

$$
\operatorname{Tr}\left(Y \lambda^{8}\right) / T_{\square}=\sqrt{3} c,
$$

where the index $T_{\square}$ appears due to our normalization $\operatorname{Tr}\left(\lambda^{i} \lambda^{j}\right)=\delta^{i j} / 2$. The confining group, $\mathrm{SU}(3)_{b}$, produces an octet of Goldstone bosons, four of which are accounted for by bachelor doublets of hypercharge $y_{D}=3 c / 2$, and from which we identify the interesting cases $c=1$ and $c=1 / 3$ which result in doubly and singly charged bachelors, respectively. Assuming an $a \leftrightarrow b$ symmetry as above, such that the SUSY breaking $D$-terms of each sector are equal, we conclude the following ratios for SM gaugino masses for cases indicated by the EM charge of their bachelor doublets:

$$
\begin{aligned}
& \text { bino: wino: gluino }\left.\right|_{Q_{D}= \pm 1}=1: 3.2: 5.2, \\
& \text { bino: wino: gluino }\left.\right|_{Q_{D}= \pm 2}=1: 1.05: 1.75 .
\end{aligned}
$$

In the interest of maintaining perturbative unification, we consider also the case of trinification, where the SM is embedded into $S U(3)^{3}$. Here we would require three copies of the gauge $\times$ global structure $\mathrm{SU}(3)_{i} \times \mathrm{SU}(3)_{i, L} \times$ $\mathrm{SU}(3)_{i, R}$, and identify hypercharge as

$$
Y=-\frac{1}{\sqrt{3}}\left(\lambda_{2 V}^{8}+\lambda_{3 V}^{8}\right)-\lambda_{3 V}^{3}
$$

with subscripts $(i, V)$ denoting generators of the diagonal subgroup of $\mathrm{SU}(3)_{i, L} \times \mathrm{SU}(3)_{i, R}$. In this case there are three singlet mesons (originating from the $\lambda_{2 V, 3 V}^{8}$ and $\lambda_{3 V}^{3}$ adjoint directions) that couple to the bino. Taking the pertinent traces as in Eq. (5.20), we find a mass matrix with two degenerate eigenvalues indicating the two components of the Dirac bino, along with two zero eigenvalues corresponding to singlet bachelors. Specifically we find eigenvalues with coefficient $\sqrt{5 / 3}$, indicating a universal gaugino spectrum up to differences in gauge couplings. With this, the predicted mass ratios become

$$
\text { bino: wino: } \text { gluino }=1: 1.5: 2.4 \text {. }
$$

We see that there is sizable variability for the bino mass relative to the other SM gauginos. The connection between anomalies and the Dirac mass can be quite enlightening here. While the gluino and wino masses must-at some level-arise from a breaking of a flavor symmetry where the diagonal component is identified with the SM gauge group, the same does not hold for the bino. Indeed, any global symmetry $\mathrm{U}(1)_{X}$ that is spontaneously broken and contains a B-Y-X anomaly will yield a Dirac mass mixing
$\pi_{X}$ and the bino. For instance, one could supplement the perturbative models we have described with additional messengers, with a spontaneously generated mass which is flavor diagonal from the outset. The axion of that spontaneous breaking would then marry the bino. Ultimately, there is one linear combination of fields that will marry the bino, and the important point is that its mass might be quite different from that expected from naive ideas of unification. For instance, if one considers a high scale model, with a large number of Goldstone fields, one could end up with a surprisingly massive bino, while still maintaining an expected GUT relation between the gluino and wino.

Of course, this all assumes we insist on maintaining some embedding within a simple group. We can also consider situations where near the Planck scale we have the classic supersoft operators generated for gluino, bino, and wino, but with no bachelor fields and only adjoints. Alternatively, it may be that only the gluino has an adjoint partner, such as in $[11,13]$, with slightly smaller Majorana masses for the gauginos, and soft masses for the scalars as in the MSSM. Such a possibility could arise with the Dirac gluino mass generated either from high scale physics or from an intermediate $S U(3)$ SUSY QCD theory as we have described. In these cases, the unification of couplings in the MSSM would be an accident. Any of these possibilities [trinification, embedding in SU(3), gluino only, bachelorfree] are reasonable boundary conditions to consider for Goldstone gaugino models, as are no doubt many others.

Finally, we note that at least some GUT scenarios predict the existence of extra doublets with hypercharge $\pm 1 / 2$ as part of complete GUT multiplets [either bachelors in $S U(3)^{3}$ or the $3-3-3-2-1$ model we have described above]. If present, this allows the possibility of a "sister Higgs" NMSSM [77], ${ }^{12}$ which produces a Type-I two Higgs doublet model (2HDM) model at low energies, which is far less constrained than the Type-II 2HDM at low $\tan \beta$.

\section{Basic phenomenology}

Many beyond the standard model (BSM) searches are sensitive to models with Dirac gauginos, but the potential suppression of the gaugino signal and the presence of new scalar states can change the focus of these searches. In particular, colored sgluons $s_{c}, \pi_{c}$ are likely to be a major discovery channel at hadron colliders. In all of the models discussed the classic supersoft operator (2.1) is generated at one-loop. This leads to the desired Dirac gaugino masses $\left(m_{D}\right)$ and also soft masses for the scalar sgluons $s_{c}, m_{s_{c}}=$ $2 m_{D}$ [14]. But since the lemon-twist operator (2.2) is not generated, the pseudoscalar Goldstone sgluons (or psgluons) $\pi_{c}$ remain massless at that order. The usual

\footnotetext{
${ }^{12}$ Similar fields, called "auxiliary Higgses" in the setup of induced electroweak symmetry breaking [78], may also play a role in this area of model building.
} 
supersoft mechanism which generates squark masses similarly leads to psgluon squared masses $m_{\pi_{c}}^{2}=$ $3 \alpha_{3} m_{D}^{2} \ln (4) / \pi$ [14]. This leads to the generic expectation that the psgluon masses are comparable to the squark masses $\left(m_{\pi_{c}}=1.5 \times m_{\tilde{q}}\right)$ and are a factor $\sim 3$ suppressed relative to the Dirac gauginos.

The collider phenomenology of sgluons at the LHC has been considered extensively [22,24,27,79-96]. We will focus on the lightest colored sparticles, which are the psgluons and the squarks. The psgluons are neutral under any $R$-symmetry and couple to gluons at tree-level through standard QCD interactions and pairs of squarks at one-loop. They also couple at one-loop to quark-antiquark pairs with coupling proportional to the quark masses.

At hadron colliders the psgluons may be pair produced at tree level from gluon initiated processes, $g g \rightarrow \pi_{c} \pi_{c}$ (dominant), or singly produced at one-loop from gluon and quark initial states, $g g, q \bar{q} \rightarrow \pi_{c}$ (subdominant). The pair production mode is the most promising for collider searches [22]. If the masses for psgluons and squarks are dominated by the radiative contributions we have discussed, the psgluons are kinematically forbidden from decaying to squarks (recalling that $m_{\pi_{c}}=1.5 \times m_{\tilde{q}}$ ). They dominantly decay directly to $q \bar{q}$ pairs. A number of searches for dijet decays of scalar octets have been performed at the LHC [88] and the (model-dependent) limit on sgluon masses could be as high as $\sim 750 \mathrm{GeV}$ with $20 \mathrm{fb}^{-1}$ of data at $\sqrt{s}=8 \mathrm{TeV}$ for decays to a pair of top quarks [96]. Taking this value for the psgluon mass as a proxy would then imply $m_{D 3} \gtrsim 2 \mathrm{TeV}$, for which the tuning of the electroweak scale is still quite mild. Searches at LHC14 will extend the reach substantially.

Unlike sgluons, psgluons have no trilinear coupling to squarks, and instead decay to a pair of quarks through gluino loops. The widths are proportional to the masses of the final state quarks, and thus psgluon pair production would likely result in top-rich final states. Interestingly, a recent excess in four-top final states has been reported by the ATLAS Collaboration [97]. This excess can be interpreted in terms of a scalar octet with mass $\approx 830 \mathrm{GeV}$, decaying with a $100 \%$ branching ratio to $t \bar{t}$. The resulting scales are naturally accommodated in the minimal GoGa setup and suggest that the (Dirac) gluino mass should be $m_{D 3} \approx 2.5 \mathrm{TeV}$ and that stops should be present below a $\mathrm{TeV}$, providing a straightforward prediction of the minimal model if such an excess persists in future data.

If there are modifications to the radiative mass contributions discussed in this work, Goldstone psgluons can decay to pairs of squarks. In this case the relevant final state involves four squarks which may then decay to neutral lightest superpartner states via $R$-parity preserving channels or potentially to SM final states via small $R$-parity violating interactions. Any search for these decays is heavily dependent on the spectrum of electroweak states and on the magnitude of various couplings; thus it is model dependent.
It is not the goal of this work to perform a dedicated collider study. Nonetheless, it is clear that Goldstone gaugino models lead to interesting collider signatures which are discoverable at the LHC.

\section{CONCLUSIONS}

The absence of any sign of low scale SUSY at the LHC compels us to consider more tuned models or models that qualitatively differ from the MSSM. Models with Dirac gauginos provide an intriguing possibility of a qualitatively different BSM scenario. In particular, the radiative corrections yield different spectra and potentially improved naturalness of the electroweak scale compared with the MSSM.

Unfortunately, models of Dirac gauginos generically have tachyons, and previously considered means to remove them have spoiled many of the appealing features of the models. The recently proposed GoGa mechanism provides a simple realization of Dirac gauginos that is free of charged tachyons in the spectrum. This allows us to both engage in producing simple models of GoGas, but also to study implications of high scale models where the lemontwist operator is absent as a boundary condition.

This simple fact-that the tachyon is absent with Goldstone gauginos, together with the simple models that realize this - puts Dirac gaugino models on a firmer footing and helps make them once again a viable candidate for a natural model of the weak scale.

\section{ACKNOWLEDGMENTS}

We thank Nathaniel Craig, Sergei Dubovsky, Claudia Frugiuele, Brian Henning, Anson Hook, Patrick Fox, Adam Martin, John Terning, and Jay Wacker for useful discussions. D. A. is supported by NSF-PHY-0969510 (the LHC Theory Initiative). N. W. and D. A. are supported by the NSF under Grants No. PHY-0947827 and No. PHY-1316753. J. G. is supported by the James Arthur Postdoctoral Fellowship at NYU, and thanks the Aspen Center for Physics (National Science Foundation Grant No. PHYS1066293) for hospitality during this work. M. M. acknowledges support from a CERN COFUND Fellowship.

\section{APPENDIX A: ABSENCE OF LTS-A DIAGRAMMATIC PROOF}

The absence of lemon twist in the GoGa setup can be proven in a simple diagrammatic way, which we present here. The essential point is to show that the interactions between messengers and adjoints, which allow the construction of pseudoscalar mass terms in the conventional Dirac gaugino case, are precisely canceled once we account for higher order terms in the Goldstone expansion of the superpotential.

The lemon-twist operator is generated when messengers are integrated out from a combination of loops constructed from either trilinear or quartic couplings. In particular, the mass of $a_{R}$ receives corrections from loops with trilinear 
and quartic terms while the mass of $a_{I}$ is generated only via quartic interactions. The shift symmetry of the GoGa model then must produce a cancellation of the quartic interactions involving $a_{I}$, and this is instructive to view diagrammatically. Keeping the messenger auxiliary fields $F_{T, \bar{T}}$ explicit in the diagrams in order to illustrate differences between the superpotentials, we have scalar interactions such as

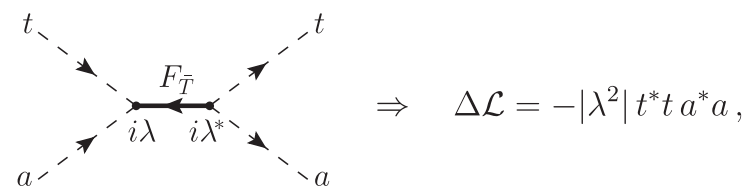

with an analogous term involving $\bar{t}$ from taking $T \leftrightarrow \bar{T}$. Closing the $t / \bar{t}$ line generates a mass at order $D^{2}$ for both the real and the imaginary components of $a$,

$$
\delta m_{a_{R, I}}^{2}=+\frac{\lambda^{2}}{16 \pi^{2}} \frac{D^{2}}{m_{T}^{2}},
$$

where $m_{T}$ is the supersymmetric $T \bar{T}$ mass, identified as $m_{T} \rightarrow \lambda f$ in the GoGa case. The trilinear coupling of the real component generates an additional one-loop graph giving

$$
\delta m_{a_{R}}^{2}=-\frac{\lambda^{2}}{8 \pi^{2}} \frac{D^{2}}{\mu^{2}},
$$

and thus $\delta m_{a_{R}}^{2}<0$ in total, signaling the problematic instability.

In the GoGa model, we must account for the new superpotential terms that arise from expanding $\exp (A / f)$ beyond linear order. At quadratic order in $A$ the crucial term is $\Delta W=\lambda A^{2} T \bar{T} / 2 f$, generating

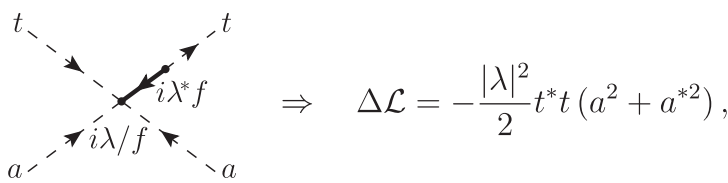

again with analogous $\bar{t}$ terms. The combination of the terms in (A1) and (A4) now gives a vanishing $t^{*} t a_{I}^{2}$ interaction, guaranteeing that no soft mass for $a_{I}$ is generated (and hence no lemon twist) since this is the only interaction feeding into the pseudoscalar soft mass once the messengers are integrated out.

The real scalar retains cubic and quartic interactions with the messengers, but the latter is modified in such a way that the two types of loop contributions to $m_{a_{R}}^{2}$ precisely cancel. In general the part of the radiative correction to the real scalar's two-point function which is independent of the SM gauge couplings (which is all that is relevant for lemon twist) is given by

$$
\delta \Sigma_{a_{R}}^{(2)}=\frac{1}{8 \pi^{2}}\left(c_{3}^{2}-m_{T}^{2} c_{4}\right) \frac{D^{2}}{m_{T}^{4}}
$$

in the presence of cubic $\left(c_{3}\right)$ and quartic $\left(c_{4}\right)$ couplings to messengers. In the conventional toy model $c_{3}=2 \lambda m_{T}$ and $c_{4}=2 \lambda^{2}$. In the GoGa model the additional interaction (A4) contributes with $\delta c_{4}=2 m_{T} \lambda / f$, which upon inserting the messenger mass $m_{T}=\lambda f$ gives a complete cancellation between the two terms entering (A5) and indicates the absence of an LTS contribution to $m_{a_{R}}^{2}$.

The shift symmetry prevents the generation of a pseudoscalar mass which is independent of the SM gauge couplings, so the above cancellations come as no surprise. But looking directly at the interactions and their modified versions in the GoGa model shows where and how these cancellations emerge in an illustrative way.

Meanwhile, there is no difference between the radiative generation of Dirac gaugino masses in the conventional and GoGa mechanisms. Thus, while we still get the same classic supersoft operator, the lemon-twist operator is absent.

\section{APPENDIX B: ABSENCE OF LTS-CONNECTION TO HOLOMORPHIC GAUGE COUPLING}

Another way to explicitly check that the lemontwist operator is not generated in the GoGa toy model discussed in Sec. III A is by looking at the renormalization of the $U(1)_{T}$ holomorphic gauge coupling. Integrating out the messengers corrects the $U(1)_{T}$ holomorphic gauge coupling below the messenger threshold, and could generate lemon-twist type operators [52]. In particular, a $\Pi^{2}$-lemon-twist operator

$$
W_{\mathrm{LTS}} \propto \frac{1}{f^{2}} \operatorname{Tr}\left[\Pi^{2}\right] W_{T}^{\alpha} W_{T \alpha}
$$

can be generated only if the messengers' masses are corrected when a $\Pi$ background superfield is turned on. Recalling that the superpotential in the messenger sector is given by

$$
W=\lambda \bar{T} \Sigma T,
$$

and turning on a $\Sigma$ background superfield, we can integrate out $\bar{T}, T$ at $m_{T}=\lambda \Sigma$. The one-loop holomorphic gauge coupling is then

$$
\tau_{1 \text {-loop }}=\frac{4 \pi i}{g^{2}(\Lambda)}+\frac{i b_{L}}{\pi} \ln \frac{\mu}{\Lambda}-\frac{i}{\pi} \ln \frac{\operatorname{Det}(\lambda \Sigma)}{\Lambda^{F}},
$$

where $b_{L}$ is the $\mathrm{U}(1)_{T} \beta$-function coefficient below the $m_{T}$ threshold. Analytically continuing $\Sigma$ into superspace, we infer that the following operator is generated at low energies:

$\frac{1}{16 \pi^{2}} \operatorname{Tr} \log \frac{\lambda \Sigma}{\Lambda} W_{T}^{\alpha} W_{T \alpha}=\frac{F}{16 \pi^{2}} \log \frac{\lambda(v+\sigma)}{\Lambda} W_{T}^{\alpha} W_{T \alpha}$,

where we have used $\Sigma=(v+\sigma) e^{\Pi^{a} T^{a} / f}$ and the fact that the generators $T^{a}$ are traceless. 
The operator (B4) is independent of the Goldstone superfields $\Pi$, so the $\Pi^{2}$-lemon-twist operator (B1) is not generated at one-loop. Moreover, because the one-loop holomorphic gauge coupling is exact, $\Pi^{2}$-lemon-twist is not generated at any order in perturbation theory.

\section{APPENDIX C: ABSENCE OF LTS-TADPOLE SHIFT IN LINEAR REPRESENTATION}

As we have discussed, there are a variety of means to achieve the appropriate breaking of the UV global symmetry required by the GoGa mechanism, including a range of both renormalizable and nonrenormalizable perturbative models. If we treat the Goldstone gaugino nonlinearly, i.e., as pions $v e^{\Pi / f}$, the protection against the lemon-twist operator is manifest [60]. However, we can also work with fields expanded linearly around the vacuum, in which case the cancellation is not as obvious. It can be instructive to see how this occurs in those cases.

As we described in Sec. III, a simple parametrization of the global symmetry breaking dynamics is given by

$$
W=\frac{1}{\Lambda^{N-2}} S\left(\operatorname{Det} \Sigma-v^{N}\right) .
$$

As noted previously, this approximates the dynamics of SUSY QCD with an equal number of colors and flavors $(F=N)$. In the vacuum, the symmetry breaking structure is $\mathrm{SU}(N)_{L} \times \mathrm{SU}(N)_{R} \rightarrow \mathrm{SU}(N)_{V}$ as the $F$-term for $S$ requires a vacuum expectation value $\langle\Sigma\rangle=v \mathbf{1}_{N}$. The $N^{2}-$ 1 traceless components of $\Sigma$ become Goldstone superfields of the spontaneously broken symmetry.

In order to accommodate gluinos, a gauge group $N \geq 3$ is required, meaning that these models are not renormalizable and must be UV completed at a scale $\Lambda$. Nonetheless, they provide a straightforward implementation of the Goldstone gaugino scenario and are worth considering further. We will focus on the case $N=3$, which is appropriate for a model of Dirac gluinos. If we expand out the superfield $\Sigma$ into its trace component $v+X$ (where $X$ is the perturbation of the trace around the VEV) and the traceless component $a$, i.e., $\Sigma=v+X+a$, then in the vacuum the superpotential Eq. (C1) becomes

$W=\frac{1}{\Lambda} S\left(\operatorname{Det}(a)-\frac{1}{2}(v+X) \operatorname{Tr}\left[a^{2}\right]+(v+X)^{3}-v^{3}\right)$.

$X$ has paired up with $S$ to obtain a Dirac mass $M_{X}=$ $3 v^{2} / \Lambda$. The Goldstone superfield, $a$, remains massless.

If $\Sigma$ is coupled to the SUSY breaking messengers as in (3.1), then as explained in Sec. II a $B \mu$ term for $a$ should be generated at one-loop. As described in Appendix B, we can think of the generation of this lemon-twist operator as a correction to the $\mathrm{U}(1)_{T}$ holomorphic gauge coupling. This arises because the messengers have their masses corrected in the presence of $\Sigma$ background fields. This correction enters linearly on $X$, but only at $\mathcal{O}\left(a^{2}\right)$ in $a$ (since $\operatorname{Tr}[a]=0$ ). Hence the lemon-twist term to leading order is given by

$$
W \supset-\frac{1}{32 \pi^{2} v^{2}} W^{\prime \alpha} W_{\alpha}^{\prime}\left(-2 N_{f} v X+\operatorname{Tr}\left[a^{2}\right]\right),
$$

where $2 N_{f}=6$ in this case under consideration. We ignore the $\mathcal{O}\left(X^{2}\right)$ term under the assumption that the lemon-twist corrections to the mass of $X$ are small, i.e., $D^{2} / v \ll 3 v^{2} / \Lambda$. This results in the $B \mu$ term for $a$ in the scalar potential,

$$
V \supset \frac{1}{2} m_{\mathrm{LT}}^{2} \operatorname{Tr}\left[a^{2}\right]+\text { H.c. }
$$

where $m_{\mathrm{LT}}^{2}=D^{2} / 16 \pi^{2} v^{2}$. In addition to this, a tadpole term for the heavy singlet scalar is also generated

$$
V \supset-3 v m_{\mathrm{LT}}^{2} X+\text { H.c. }
$$

When the scalar potential is minimized, this leads to an additional contribution to the symmetry breaking VEV,

$$
\langle X\rangle=\frac{3 v m_{\mathrm{LT}}^{2}}{M_{X}^{2}}=\frac{m_{\mathrm{LT}}^{2} \Lambda^{2}}{3 v^{3}} .
$$

Inserting this shifted vacuum into the $F$-term equation for $S$, to lowest order in $m_{\mathrm{LT}}^{2}$ we have

$\mathbf{F}_{\mathbf{S}}=\frac{1}{\Lambda}\left(\operatorname{Det}(a)-\frac{1}{2}\left(v+\frac{m_{L T}^{2} \Lambda^{2}}{3 v^{3}}\right) \operatorname{Tr}\left[a^{2}\right]+\frac{m_{\mathrm{LT}}^{2} \Lambda^{2}}{v}\right)$.

Squaring this $F$-term to solve for the scalar potential we find that the dangerous $B \mu$ term of Eq. (C4) is exactly canceled. This is not surprising - the symmetries alone dictate this, and in the nonlinear realization it is manifest. Working with linear fields around the origin, however, this is much more involved: in this formulation the absence of $B \mu$ terms occurs through cancellations of the explicit mass terms generated from messenger loops against new $B \mu$ terms generated by a shift in the VEV of a heavy field in the symmetry breaking sector. Such a scenario shows clearly that such cancellations can occur in a very nontrivial fashion when the Goldstone nature of the right-handed gaugino is obscured. 
[1] J. D. Wells, Implications of supersymmetry breaking with a little hierarchy between gauginos and scalars, arXiv:hep-ph/ 0306127.

[2] N. Arkani-Hamed and S. Dimopoulos, Supersymmetric unification without low energy supersymmetry and signatures for fine-tuning at the LHC, J. High Energy Phys. 06 (2005) 073.

[3] G. Giudice and A. Romanino, Split supersymmetry, Nucl. Phys. B699, 65 (2004).

[4] P. J. Fox, D. E. Kaplan, E. Katz, E. Poppitz, V. Sanz, M. Schmaltz, M. D. Schwartz, and N. Weiner, Supersplit supersymmetry, arXiv:hep-th/0503249.

[5] A. Arvanitaki, N. Craig, S. Dimopoulos, and G. Villadoro, Mini-Split, J. High Energy Phys. 02 (2013) 126.

[6] N. Arkani-Hamed, A. Gupta, D. E. Kaplan, N. Weiner, and T. Zorawski, Simply unnatural supersymmetry, arXiv: 1212.6971.

[7] P. Fayet, Massive gluinos, Phys. Lett. 78B, 417 (1978).

[8] J. Polchinski and L. Susskind, Breaking of supersymmetry at intermediate energy, Phys. Rev. D 26, 3661 (1982).

[9] L. Girardello, M. Porrati, and A. Zaffaroni, Spontaneously broken $N=2$ supergravity without light mirror fermions, Nucl. Phys. B505, 272 (1997).

[10] N. Polonsky and S. Su, Low-energy limits of theories with two supersymmetries, Phys. Rev. D 63, 035007 (2001).

[11] L. Hall and L. Randall, U(1)-R symmetric supersymmetry, Nucl. Phys. B352, 289 (1991).

[12] L. Randall and N. Rius, The minimal U(1)-R symmetric model revisited, Phys. Lett. B 286, 299 (1992).

[13] A. E. Nelson, N. Rius, V. Sanz, and M. Unsal, The minimal supersymmetric model without a mu term, J. High Energy Phys. 08 (2002) 039.

[14] P. J. Fox, A. E. Nelson, and N. Weiner, Dirac gaugino masses and supersoft supersymmetry breaking, J. High Energy Phys. 08 (2002) 035.

[15] G. D. Kribs and A. Martin, Supersoft supersymmetry is super-safe, Phys. Rev. D 85, 115014 (2012).

[16] G. D. Kribs, E. Poppitz, and N. Weiner, Flavor in supersymmetry with an extended R-symmetry, Phys. Rev. D 78, 055010 (2008).

[17] I. Antoniadis, A. Delgado, K. Benakli, M. Quiros, and M. Tuckmantel, Splitting extended supersymmetry, Phys. Lett. B 634, 302 (2006).

[18] I. Antoniadis, K. Benakli, A. Delgado, M. Quiros, and M. Tuckmantel, Split extended supersymmetry from intersecting branes, Nucl. Phys. B744, 156 (2006).

[19] I. Antoniadis, K. Benakli, A. Delgado, and M. Quiros, A new gauge mediation theory, Adv. Stud. Theor. Phys. 2, 645 (2008).

[20] S. D. L. Amigo, A. E. Blechman, P. J. Fox, and E. Poppitz, R-symmetric gauge mediation, J. High Energy Phys. 01 (2009) 018.

[21] A.E. Blechman, R-symmetric gauge mediation and the minimal R-symmetric supersymmetric standard model, Mod. Phys. Lett. A 24, 633 (2009).

[22] T. Plehn and T. M. Tait, Seeking sgluons, J. Phys. G 36, 075001 (2009).

[23] K. Benakli and M. Goodsell, Dirac gauginos in general gauge mediation, Nucl. Phys. B816, 185 (2009).
[24] S. Choi, M. Drees, J. Kalinowski, J. Kim, E. Popenda, and P. M. Zerwas, Color-octet scalars of $N=2$ supersymmetry at the LHC, Phys. Lett. B 672, 246 (2009).

[25] G. Belanger, K. Benakli, M. Goodsell, C. Moura, and A. Pukhov, Dark matter with Dirac and Majorana gaugino masses, J. Cosmol. Astropart. Phys. 08 (2009) 027.

[26] K. Benakli and M. Goodsell, Dirac gauginos and kinetic mixing, Nucl. Phys. B830, 315 (2010).

[27] S. Choi, J. Kalinowski, J. Kim, and E. Popenda, Scalar gluons and Dirac gluinos at the LHC, Acta Phys. Pol. B 40, 2913 (2009).

[28] K. Benakli and M. Goodsell, Dirac gauginos, gauge mediation and unification, Nucl. Phys. B840, 1 (2010).

[29] S. Choi, D. Choudhury, A. Freitas, J. Kalinowski, J. Kim, and P. M. Zerwas, Dirac neutralinos and electroweak scalar bosons of $N=1 / N=2$ hybrid supersymmetry at colliders, J. High Energy Phys. 08 (2010) 025.

[30] L. M. Carpenter, Dirac gauginos, negative supertraces and gauge mediation, J. High Energy Phys. 09 (2012) 102.

[31] G. D. Kribs, T. Okui, and T. S. Roy, Viable gravity-mediated supersymmetry breaking, Phys. Rev. D 82, 115010 (2010).

[32] S. Abel and M. Goodsell, Easy Dirac gauginos, J. High Energy Phys. 06 (2011) 064.

[33] R. Davies, J. March-Russell, and M. McCullough, A supersymmetric one Higgs doublet model, J. High Energy Phys. 04 (2011) 108.

[34] K. Benakli, M. D. Goodsell, and A.-K. Maier, Generating mu and Bmu in models with Dirac gauginos, Nucl. Phys. B851, 445 (2011).

[35] J. Kalinowski, Phenomenology of R-symmetric supersymmetry, Acta Phys. Pol. B 42, 2425 (2011).

[36] C. Frugiuele and T. Gregoire, Making the sneutrino a Higgs with a $U(1)_{R}$ lepton number, Phys. Rev. D 85, 015016 (2012).

[37] H. Itoyama and N. Maru, D-term dynamical supersymmetry breaking generating split $N=2$ gaugino masses of mixed Majorana-Dirac type, Int. J. Mod. Phys. A 27, 1250159 (2012).

[38] H. Itoyama and N. Maru, D-term triggered dynamical supersymmetry breaking, Phys. Rev. D 88, 025012 (2013).

[39] H. Itoyama and N. Maru, $126 \mathrm{GeV}$ Higgs boson sssociated with D-term triggered dynamical supersymmetry breaking, Symmetry 7, 193 (2015).

[40] K. Rehermann and C. M. Wells, Weak scale leptogenesis, R-symmetry, and a displaced Higgs, arXiv:1111.0008.

[41] E. Bertuzzo and C. Frugiuele, Fitting neutrino physics with a $U(1)_{R}$ lepton number, J. High Energy Phys. 05 (2012) 100.

[42] R. Argurio, M. Bertolini, L. Di Pietro, F. Porri, and D. Redigolo, Holographic correlators for general gauge mediation, J. High Energy Phys. 08 (2012) 086.

[43] M. D. Goodsell, Two-loop RGEs with Dirac gaugino masses, J. High Energy Phys. 01 (2013) 066.

[44] R. Fok, G. D. Kribs, A. Martin, and Y. Tsai, Electroweak baryogenesis in R-symmetric supersymmetry, Phys. Rev. D 87, 055018 (2013).

[45] R. Argurio, M. Bertolini, L. Di Pietro, F. Porri, and D. Redigolo, Exploring holographic general gauge mediation, J. High Energy Phys. 10 (2012) 179. 
[46] C. Frugiuele, T. Gregoire, P. Kumar, and E. Ponton, " $L=R "-U(1)_{R}$ as the origin of leptonic "RPV", J. High Energy Phys. 03 (2013) 156.

[47] C. Frugiuele, T. Gregoire, P. Kumar, and E. Ponton, " $L=R$ " $-U(1)_{R}$ lepton number at the LHC, J. High Energy Phys. 05 (2013) 012.

[48] K. Benakli, M. D. Goodsell, and F. Staub, Dirac gauginos and the $125 \mathrm{GeV}$ Higgs, J. High Energy Phys. 06 (2013) 073.

[49] G. D. Kribs and A. Martin, Dirac gauginos in supersymmetry-Suppressed jets + MET signals: A Snowmass Whitepaper, arXiv:1308.3468.

[50] G. D. Kribs and N. Raj, Mixed gauginos sending mixed messages to the LHC, Phys. Rev. D 89, 055011 (2014).

[51] S. Chakraborty and S. Roy, Higgs boson mass, neutrino masses and mixing and keV dark matter in an $U(1)_{R}$ lepton number model, J. High Energy Phys. 01 (2014) 101.

[52] C. Csaki, J. Goodman, R. Pavesi, and Y. Shirman, The $m_{D}-$ $b_{M}$ problem of Dirac gauginos and its solutions, Phys. Rev. D 89, 055005 (2014).

[53] H. Beauchesne and T. Gregoire, Electroweak precision measurements in supersymmetric models with a $\mathrm{U}(1)_{R}$ lepton number, J. High Energy Phys. 05 (2014) 051.

[54] E. Bertuzzo, C. Frugiuele, T. Gregoire, and E. Ponton, Dirac gauginos, R symmetry and the $125 \mathrm{GeV}$ Higgs, J. High Energy Phys. 04 (2015) 089.

[55] K. Benakli, M. Goodsell, F. Staub, and W. Porod, Constrained minimal Dirac gaugino supersymmetric standard model, Phys. Rev. D 90, 045017 (2014).

[56] S. Dimopoulos, K. Howe, and J. March-Russell, Maximally Natural Supersymmetry, Phys. Rev. Lett. 113, 111802 (2014).

[57] S. Chakraborty, D. K. Ghosh, and S. Roy, $7 \mathrm{keV}$ Sterile neutrino dark matter in $U(1)_{R}$ lepton number model, J. High Energy Phys. 10 (2014) 146.

[58] A. E. Nelson and T. S. Roy, New Supersoft Supersymmetry Breaking Operators and a Solution to the $\mu$ Problem, Phys. Rev. Lett. 114, 201802 (2015).

[59] L. M. Carpenter and J. Goodman, New calculations in Dirac gaugino models: Operators, expansions, and effects, J. High Energy Phys. 07 (2015) 017.

[60] D. S. M. Alves, J. Galloway, M. McCullough, and N. Weiner, Goldstone Gauginos, Phys. Rev. Lett. 115, 161801 (2015).

[61] A. Arvanitaki, M. Baryakhtar, X. Huang, K. van Tilburg, and G. Villadoro, The last vestiges of naturalness, J. High Energy Phys. 03 (2014) 022.

[62] D. S. M. Alves and J. Galloway, Wess-Zumino-Witten terms and Dirac gauginos (to be published).

[63] N. Seiberg, Electric-magnetic duality in supersymmetric non-Abelian gauge theories, Nucl. Phys. B435, 129 (1995).

[64] K. A. Intriligator and N. Seiberg, Lectures on supersymmetric gauge theories and electric-magnetic duality, Nucl. Phys. B, Proc. Suppl. 45, 1 (1996).

[65] N. Arkani-Hamed, G. F. Giudice, M. A. Luty, and R. Rattazzi, Supersymmetry breaking loops from analytic continuation into superspace, Phys. Rev. D 58, 115005 (1998).
[66] N. Arkani-Hamed and R. Rattazzi, Exact results for nonholomorphic masses in softly broken supersymmetric gauge theories, Phys. Lett. B 454, 290 (1999).

[67] A. Manohar and H. Georgi, Chiral quarks and the nonrelativistic quark model, Nucl. Phys. B234, 189 (1984).

[68] J. Wess and B. Zumino, Consequences of anomalous Ward identities, Phys. Lett. 37B, 95 (1971).

[69] E. Witten, Global aspects of current algebra, Nucl. Phys. B223, 422 (1983).

[70] J. R. Espinosa and M. Quiros, Gauge Unification and the Supersymmetric Light Higgs Mass, Phys. Rev. Lett. 81, 516 (1998).

[71] R. Barbieri, L. J. Hall, A. Y. Papaioannou, D. Pappadopulo, and V. S. Rychkov, An alternative NMSSM phenomenology with manifest perturbative unification, J. High Energy Phys. 03 (2008) 005.

[72] D. S. M. Alves, J. Liu, and N. Weiner, Hiding missing energy in missing energy, J. High Energy Phys. 04 (2015) 088.

[73] T. Moroi, H. Murayama, and T. Yanagida, The Weinberg angle without grand unification, Phys. Rev. D 48, R2995 (1993).

[74] B. Brahmachari, U. Sarkar, and K. Sridhar, Nonperturbative unification in the light of LEP results, Mod. Phys. Lett. A 08, 3349 (1993).

[75] V. Rizov, A gauge model of the electroweak and strong interactions based on the group $\mathrm{SU}(3)_{L} \times \mathrm{SU}(3)_{R} \times \mathrm{SU}(3)_{c}$, Bulgarian Journal of Physics 8, 461 (1981).

[76] A. de Rújula, H. Georgi, and S. L. Glashow, in Proceedings of the Fifth Workshop on Grand Unification, edited by K. Kang, H. Fried, and P. Frampton (World Scientific, Singapore, 1984), p. 88.

[77] D. S. Alves, P. J. Fox, and N. Weiner, Supersymmetry with a sister Higgs, Phys. Rev. D 91, 055003 (2015).

[78] J. Galloway, M. A. Luty, Y. Tsai, and Y. Zhao, f electroweak symmetry breaking and supersymmetric naturalness, Phys. Rev. D 89, 075003 (2014).

[79] S. Choi, M. Drees, J. Kalinowski, J. Kim, E. Popenda, and P. M. Zerwas, Color-octet scalars at the LHC, Acta Phys. Pol. B 40, 1947 (2009).

[80] A. Idilbi, C. Kim, and T. Mehen, Factorization and resummation for single color-octet scalar production at the LHC, Phys. Rev. D 79, 114016 (2009).

[81] A. Idilbi, C. Kim, and T. Mehen, Pair production of color-octet scalars at the LHC, Phys. Rev. D 82, 075017 (2010).

[82] M. Martynov and A. Smirnov, Production of colored scalar particles in $p p$ collisions and masses of scalar gluons from future LHC data, Phys. At. Nucl. 73, 1207 (2010).

[83] T. Han, I. Lewis, and Z. Liu, Colored resonant signals at the LHC: Largest rate and simplest topology, J. High Energy Phys. 12 (2010) 085.

[84] S. Schumann, A. Renaud, and D. Zerwas, Hadronically decaying color-adjoint scalars at the LHC, J. High Energy Phys. 09 (2011) 074.

[85] G. Aad et al. (ATLAS Collaboration), Search for massive colored scalars in four-jet final states in $\sqrt{s}=7 \mathrm{TeV}$ proton-proton collisions with the ATLAS Detector, Eur. Phys. J. C 71, 1828 (2011). 


\section{MODELS OF GOLDSTONE GAUGINOS}

[86] D. Goncalves-Netto, D. Lopez-Val, K. Mawatari, T. Plehn, and I. Wigmore, Sgluon pair production to next-to-leading order, Phys. Rev. D 85, 114024 (2012).

[87] W. Kotlarski and J. Kalinowski, Scalar gluons at the LHC, Acta Phys. Pol. B 42, 2485 (2011).

[88] G. Aad et al. (ATLAS Collaboration), Search for pairproduced massive coloured scalars in four-jet final states with the ATLAS detector in proton-proton collisions at $\sqrt{s}=7$ TeV, Eur. Phys. J. C 73, 2263 (2013).

[89] ATLAS Collaboration, Search for massive coloured scalars with the ATLAS Detector in four-jet final states using 4.6 inverse $\mathrm{fb}$ of $\sqrt{s}=7 \mathrm{TeV}$ proton-proton collision data, ATLAS-CONF-2012-110.

[90] S. Calvet, B. Fuks, P. Gris, and L. Valery, Searching for sgluons in multitop events at a center-of-mass energy of $8 \mathrm{TeV}$, J. High Energy Phys. 04 (2013) 043.

[91] ATLAS Collaboration and T. A. Collaboration, Search for anomalous production of events with same-sign dileptons and $\mathrm{b}$ jets in 14.3 inverse $\mathrm{fb}$ of $p p$ collisions at $\sqrt{s}=8 \mathrm{TeV}$ with the ATLAS detector, arXiv:1310.0147.
PHYSICAL REVIEW D 93, 075021 (2016)

[92] D. Paredes, Search for new physics in events with 4 top quarks in the ATLAS detector at the LHC, Ph.D. thesis, CERN-THESIS-2013-202.

[93] C.-Y. Chen, A. Freitas, T. Han, and K. S. M. Lee, Heavy color-octet particles at the LHC, J. High Energy Phys. 05 (2015) 135.

[94] A. Renaud, Search for scalar gluons with the ATLAS detector at the LHC, Ph.D. thesis, CERN-THESIS-2012404.

[95] L. Valery, Recherche de sgluons dans des états finals multitops avec le détecteur ATLAS auprès du LHC à $\sqrt{s}=8 \mathrm{TeV}$, Ph.D. thesis, CERN-THESIS-2014-075.

[96] L. Beck, F. Blekman, D. Dobur, B. Fuks, J. Keaveney, and K. Mawatari, Probing top-philic sgluons with LHC Run I data, Phys. Lett. B 746, 48 (2015).

[97] G. Aad et al. (ATLAS Collaboration), Analysis of events with $b$-jets and a pair of leptons of the same charge in $p p$ collisions at $\sqrt{s}=8 \mathrm{TeV}$ with the ATLAS detector, J. High Energy Phys. 10 (2015) 150. 